\title{
Effective Potential Description of the Interaction between Single Stem Cells and Localized Ligands
}

\author{
Ignacio Bordeu, ${ }^{1,2,3, *, \sharp}$ Clare Garcin, ${ }^{3,8, \|}$ Shukry J. Habib, ${ }^{3, \dagger}$ and Gunnar Pruessner, ${ }^{1,2, \pi}$ \\ ${ }^{1}$ Department of Mathematics, Imperial College London, London SW7 2AZ, United Kingdom \\ ${ }^{2}$ Centre of Complexity Science and Department of Mathematics, \\ Imperial College London, SW7 2AZ, United Kingdom \\ ${ }^{3}$ Centre for Stem Cells and Regenerative Medicine, King's College London, \\ London SE1 9RT, United Kingdom
}

(Received 10 May 2020; revised 10 August 2020; accepted 10 September 2020; published 30 October 2020)

Cell signaling is essential for cell fate determination and tissue patterning. As signaling ligands are presented to the receiving cell, they are recruited and recognized by the cell membrane so as to elicit a biological response and to pattern multicellular tissues. Cells can accumulate and transport these ligands, which results in an emergent organization of the ligands' spatial distribution. To study this organization, we make use of a simplified experimental setup, in which single mouse embryonic stem cells (mESCs) can interact with immobilized ligands. We introduce a two-species age-dependent correlation function that allows the description and quantification of the spatiotemporal dynamics of single cell-ligand interactions. Through the analysis of mESC data and numerical simulations, we show that cells act as effective forcefield generators, perturbing and organizing their environment. This organization, captured in the form of an aging effective potential, is an emergent property of the population of single cells interacting with randomly distributed localized ligands.

DOI: $10.1103 /$ PhysRevX.10.041022

\section{INTRODUCTION}

Pluripotent stem cells are the building blocks of multicellular organisms. Pluripotency refers to their ability to specialize or differentiate into any of the three germ layers-endoderm, ectoderm, and mesoderm [1]. The fate of a pluripotent stem cell is determined by multiple factors. In particular, external factors, such as chemical and mechanical [2] signals, can regulate the precise outcome of the differentiation process. Concentration gradients of

\footnotetext{
*Corresponding author. ib443@cam.ac.uk

Corresponding author. shukry.habib@kcl.ac.uk

*Present address: DAMTP and The Gurdon Institute, University of Cambridge, Cambridge, United Kingdom.

${ }^{\S}$ c.garcin@warwick.ac.uk

"Present address: Centre for Mechanochemical Cell Biology, Warwick Medical School, University of Warwick, Coventry, United Kingdom.

"g.pruessner@imperial.ac.uk

Published by the American Physical Society under the terms of the Creative Commons Attribution 4.0 International license. Further distribution of this work must maintain attribution to the author(s) and the published article's title, journal citation, and DOI.
}

\author{
Subject Areas: Biological Physics, \\ Interdisciplinary Physics, Soft Matter
}

morphogens are thought to be one mechanism responsible for controlling generic developmental processes and tissue patterning [3-6]. The producing cells can transport signaling ligands on membrane protrusions to the receiving cell or secrete them into the extracellular matrix (ECM). The ligands may either diffuse or bind to proteins in the ECM and travel to receiving cells. Alternatively, secreted ligands can be actively recruited by the receiving cell via cytonemes, thin membrane extensions projecting into the extracellular matrix [7]. In some cases, signaling ligands are tethered to the membrane of the producing cell and transmitted to the receiving neighboring cell via direct cellto-cell contact [8-10], which might provide a fast and controllable mechanism for signal propagation in tissues $[3,10,11]$. Cells detect and accumulate ligands in the environment by recruiting them towards the cell membrane via cytonemes [7], which, combined with cell movement, causes a net transport of ligands, thereby organizing their environment.

It is still unclear how the mesoscopic organization of cells and thus ligands emerges from the microscopic cellligand interactions. Understanding the spatiotemporal properties and scales involved in cell-ligand interactions at the single cell level is the basis for understanding the organization of cells at the tissue level [7]. The study of specific stem cell-ligand interactions in vivo is challenging 
due to the inherent complexity of multicellular tissues, where a myriad of signaling ligands are generated and received by cells simultaneously. Thus, pinpointing the exact role of each ligand and analyzing specific cell-ligand interaction is impractical.

In recent years, there has been much interest in applying techniques from equilibrium and nonequilibrium statistical mechanics to study cell organization, tissue formation, and homeostasis at the multicellular level. Mean-field approaches from fluids [12,13], glasses [14], and nematic liquid crystals [15] have proven useful for modeling tissue growth and aging. Specifically, correlation lengths [16] and stationary cell-cell effective potentials of interactions [17] have been quantified. However, the dynamic interactions between cells and biochemical ligands at the single cell level are only partially understood.

In this work, we make use of a previously reported method by which Wnt3a (protein) ligands are covalently immobilized to the surface of micrometer-sized beads $[18,19]$. These are inert, nontoxic beads, which have an iron-oxide nucleus encased in a thin polymer layer. This layer is coupled to carboxylic acid, which, upon conversion to succenimide ester, can bind the ligand of interest. When a mouse embryonic stem cell (mESC) comes into contact with a protein-coated, biochemically active, signaling Wnt bead, the bead provides a localized source of a self-renewal signal that can promote asymmetric cell division. The Wnt-bead-proximal daughter cell remains a $\mathrm{mESC}$, while the Wnt-bead-distal cell is prone to differentiation $[18,20]$. The Wnt protein coating can be chemically modified by treatment with dithiothreitol (DTT) [19] to generate biochemically inactive, nonsignaling iWnt beads. As shown by Habib et al. [18], when a cell encounters a "biologically active" Wnt bead, it actively enriches the region of the membrane with receptors, thus increasing the membranebead bond strength. On the other hand, the iWnt protein has an altered conformation, preventing it from activating the pathways associated with asymmetric cell division. Recently, Junyent et al. have shown that mESCs produce specialized cytonemes to specifically recruit self-renewal signaling Wnt beads [7]. For this reason, we expect cells to interact differently with Wnt beads compared to iWnt beads. As a control, we make use of uncoated beads, Unc beads, which have no protein coating and would otherwise stimulate the interaction between the bead and receptors on the cell membrane. None of the beads used in the present experiments is engulfed in the cytoplasm, and they remain on the membrane surface for the duration of the experiments.

To quantify the interactions between individual cells and ligands localized on the beads' surface, we introduce a suitable correlation function. We show that cell-ligand interactions give rise to, and can be quantified by, aging effective potentials. These potentials capture cell-ligand interactions from the level of the microscopic positioning of beads up to the mesoscopic organization of the environment. They characterize the interaction strength and range and reveal how cells position ligands on the cell membrane while also shedding light on the consequences of transport of ligands by cell movement. This method reveals differences in the interaction of cells with different types of bead coatings (signaling and nonsignaling ligands, and uncoated), which affect both the spatial and temporal scales of the interactions. By performing numerical simulations of simplified models of the cell-bead system, we could identify different mechanisms in the cell-ligand interaction. Furthermore, we explore a regime where the transport of beads by cells controls a smooth transition of the effective potential from being effectively attractive, promoting the accumulation of beads, to effectively repulsive, promoting a depletion of beads around the cells. This behavior turns out to be key to reproduce the experimental observations. (a)

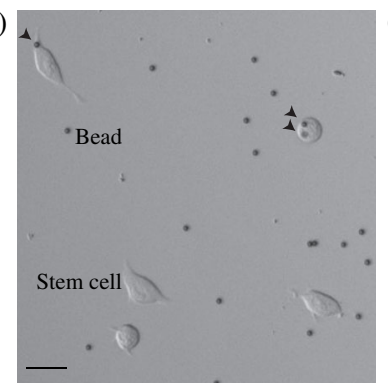

(c)

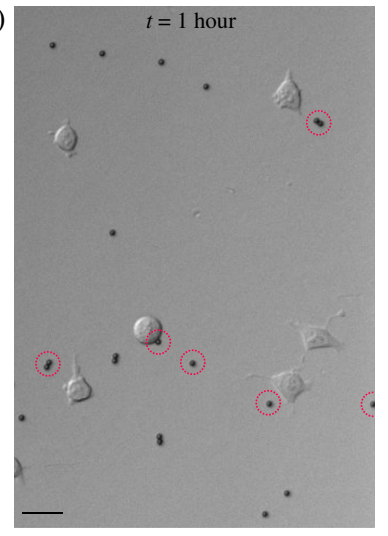

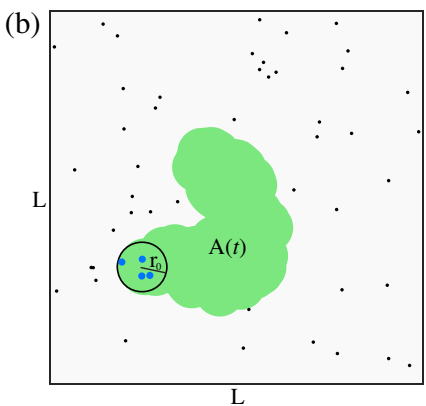

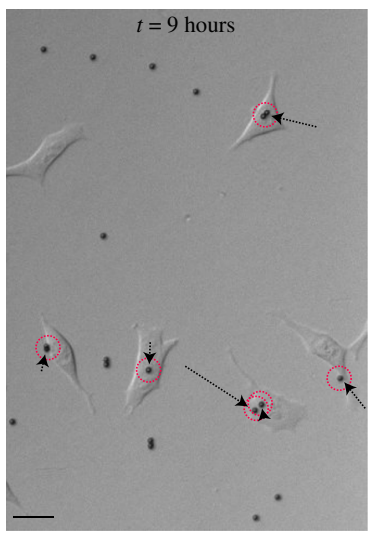

FIG. 1. (a) Snapshot of the experimental observation of single mESCs and beads. Arrowheads indicate beads in contact with cells. (b) Simulation of a single cell of radius $r_{0}=1 / 15$ (encircled black) performing a random walk with step length $\ell=r_{0} / 5$, on a periodic system of linear size $L=1$. The area covered by the cell up to time $t=500, A(t)$, is shown in green. Dots correspond to beads initially randomly distributed in the environment (black) and beads captured by a cell (blue). The total bead density is $\rho_{b}=50$. (c) Two representative images, at times $t=1 \mathrm{~h}$ (left) and $t=9 \mathrm{~h}$ (right), of the interaction of mESCs and Wnt beads. Beads that come in contact with cells are encircled, while their net translation between the two frames is depicted by dashed arrows (right). The scale bar in panels (a) and (c) is $20 \mu \mathrm{m}$. 
The aging properties of the effective cell-ligand interactions relate to the continuous and dynamic organization of the environment by cells (see Fig. 1). We quantify this organization, based on the relative position of beads with respect to cells, by measuring the effective configurational entropy of the system, which quantifies the driving of the system away from the uniform distribution of beads. Because in the absence of cells beads remain static on the bottom of the well, thermal contributions to the entropy can be neglected. Any spatiotemporal changes in the configurational entropy of the system are therefore due to the activity of cells. Here, by casting the configurational entropy of the cell-bead system solely in terms of the aging effective potential, we are able to measure, both experimentally and numerically, its temporal evolution. We characterize this entropy rate as a function of time, and as a function of the properties of the ligands, to show that relevant biochemical signals contribute to a faster and increased organization of the environment. This organization might have relevant implications in the formation of specialized microenvironments in vivo, such as the stemcell niche [21].

The remainder of this article is organized as follows: In Sec. II, we describe the experimental setup used for the cell-ligand interaction analysis. In Sec. III, we introduce the age- and time-dependent effective potential, and in Sec. IV, we present the experimental measurements. Numerical characterization of the effective potential and its interpretation in the light of the experimental observations are shown in Secs. V and VI. In Sec. VII, we show the results on the configurational entropy based on experimental and numerical data. The conclusions and outlook of our work are left for Sec. VIII.

\section{EXPERIMENTAL SETUP}

To experimentally measure the spatiotemporal interactions between single cells and beads, we performed live imaging of mouse embryonic stem cells (W4 cells [22]) in the presence of Wnt, iWnt, or Unc beads (2.8- $\mu \mathrm{m}$ diameter, Dynabeads M-270 Carboxylic acid) at 1-min time resolution for periods of 12 hours. We seeded ca. $2500 \mathrm{mESCs}$ together with ca. $20000(0.3 \mu \mathrm{g})$ of either Wnt, iWnt, or Unc beads on each of two central wells of a black, glassbottomed, 96-well plate (Greiner), in serum and Leukemia Inhibitory Factor (LIF)-containing media, which promotes self-renewal of stem cells [23]. The ten wells surrounding the two wells of interest were filled with phosphatebuffered saline (PBS) solution to reduce evaporation of the media in the cell- and bead-containing wells. On the bottom of the $136-\mathrm{mm}^{2}$ well, the bead number density is low, reaching ca. 150 beads $/ \mathrm{mm}^{2}$. The cell number density, which reaches ca. 20 cells $/ \mathrm{mm}^{2}$, is low, such that cells are observed mostly in a single cell state for the duration of the experiments.
The time-lapse imaging at 1-min resolution for $12 \mathrm{~h}$ was performed using a Zeiss-inverted Axio Imager epifluorescence microscope, equipped with a CoolSNAP HQ2 CCD camera, at $10 \times$ amplification $(\mathrm{N} / \mathrm{A}=0.3)$. Cells were allowed to settle at $37^{\circ} \mathrm{C}, 5 \% \mathrm{CO}_{2}$ for 30 min prior to imaging. The area covered by a single position of the microscope was $874.99 \times 653.73 \mu \mathrm{m}^{2}$, containing ca. 11 cells and ca. 84 beads (see Fig. 1). About 15 nonoverlapping positions were selected per well, and each experiment was repeated at least three times (biological repeats), for a total of more than 1000 cells and more than 10000 beads per condition. The experiments avoid imagining techniques that might alter the behavior of cells, such as additional genetic manipulation, molecular markers, or phototoxicity. The time-lapse images were analyzed with a custombuilt multiparticle tracking algorithm (based on MATLAB R2018b), which extracts the trajectories of both cells and beads as a function of time (see methods Sec. A 3 for details). Our imaging protocol and tracking algorithm does not allow the precise detection of the cell membrane and other features of a linear extent of less than $0.6 \mu \mathrm{m}$. It is therefore impossible to reliably and reproducibly determine when a bead (as highlighted in Fig. 2) makes or loses contact with a cell. However, the algorithm locates the cell body and its centroid reliably, as required in the measurement of spatiotemporal correlations.

Preliminary observations indicate that in the absence of cells, beads are distributed uniformly, up to effects by excluded volume, at the bottom of the well. However, in the presence of cells, the spatial distribution of beads is strongly affected by cell-bead interactions, which appear to change in time, and depend on the bead coating. After settling at the bottom of the well, cells move and interact with the surrounding beads. When contacting a bead, the bead might get captured by binding to the cells' membranes. Cell-bead interactions can be transient; beads might
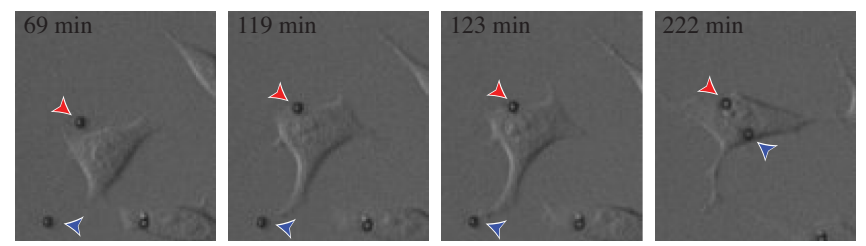

FIG. 2. mESC recruiting two Wnt beads (time $t=69,119,123$, $222 \mathrm{~min}$, increasing from left to right). These images are the raw data obtained from the microscope, which are to be fed to the tracking algorithm (methods Sec. A 3). At $t=69 \mathrm{~min}$, a bead lies by the cell (red arrowhead), yet it seems to come in contact with the cell only at $t=119$ (second frame). Subsequently, the cell extends, coming into contact with a second bead (blue arrowhead) at $t=123 \mathrm{~min}$ (third frame). This event cannot be detected reliably by the algorithm until much later, for instance, when the bead is on the cell body, $t=222 \mathrm{~min}$ (last frame). The same difficulties arise when attempting to measure the release of beads (Sec. VI). The beads are $2.8 \mu \mathrm{m}$ in diameter. 
be released, unbinding from the cell membrane and remaining at the bottom of the well. In the next section, we describe a method for quantifying cell-bead interactions and the emergence of spatial order in the bead distribution by measuring the spatiotemporal correlations of the positions of beads due to the presence of cells.

\section{AGING EFFECTIVE POTENTIAL}

The spatiotemporal structure of condensed matter has been extensively studied through the measurement of the dynamic or van Hove correlation function [24]. Its Fourier transform in space and time, namely, the dynamic structure factor, can be measured directly in X-ray experiments [24]. For mesoscopic objects, such as cells and beads, where the positions of every particle can be precisely determined, the dynamic correlation functions can be calculated in real space and time. In the cell-bead system, the cells' movement and interaction with their surroundings cause temporal changes in the correlations, known as aging [25]. It occurs because the system is initialized in a configuration that is not necessarily its steady state. Moreover, the nature of living cells might prevent the system from having a steady-state configuration in the first place, thus exhibiting permanent dynamics.

The age-dependent properties of cell-bead interaction can be quantified by the correlations in the positions of beads in relation to the positions of cells. To formalize this idea, we introduce a modified version of the van Hove correlation function, namely, a two-species age-dependent correlation function (ACF). Given a spatial arrangement of $N$ cells at positions $\mathbf{R}_{i}(t)$, and $M$ beads at positions $\mathbf{r}_{j}(t)$, we define the ACF $\hat{G}(\mathbf{r}, t, \tau)$, as a function of the position $\mathbf{r}$, age or time $t$, and delay $\tau$, as

$\hat{G}(\mathbf{r}, t, \tau)=\frac{1}{N}\left\langle\sum_{i=1}^{N} \sum_{j=1}^{M} \delta\left[\mathbf{r}-\mathbf{r}_{j}(t+\tau)+\mathbf{R}_{i}(t)\right]\right\rangle$,

where $\delta(\cdot)$ corresponds to the Dirac-delta function. This correlation function measures the density of beads at position $\mathbf{r}$ at time $t+\tau$, given that there was a cell at the origin at time $t$. The angle brackets $\langle\cdot\rangle$ indicate the ensemble average. Equation (1) corresponds to a twospecies version of the van Hove correlation function, where self-interactions are negligible, and there is an implicit dependence on time $t$, which we refer to as aging (see methods Sec. A 2 for details).

In the case of nonaging systems, i.e., without dependence on absolute time $t$, Eq. (1) depends only on the time delay $\tau$, so time $t$ can be set to zero. Hence, the ACF has a fixed value for a given delay $\tau$, independent of the moment $t$ at which the measurement has been taken. As described above, and as will be demonstrated in the next section, the ACF for cell-bead interactions does not have a fixed value for a given delay, displaying temporal evolution due to active cellular dynamics.

From the ACF, Eq. (1), we can obtain the aging paircorrelation function $\hat{g}$ from the relation

$$
\hat{G}(\mathbf{r}, t, \tau)=\rho_{b} \hat{g}(\mathbf{r}, t, \tau),
$$

where $\rho_{b}$ is the density of beads. For isotropic systems, such as our cell-bead system, the pair-correlation function depends only on the distance $r=|\mathbf{r}|$. This function is usually referred to as the radial distribution function (RDF) and is the central observable in the theory of simple liquids [24]. We then define the effective potential of interactions between the two species (cells and beads) as

$$
U^{\mathrm{eff}}(r, t, \tau)=-\ln [\hat{g}(r, t, \tau)]
$$

For systems in thermodynamic equilibrium, the effective potential is stationary in time, whereas in our setup, the active nature of cells renders the potential age dependent. An equilibrium liquid equipped with the effective potential determined by Eq. (3) will reproduce the RDF used in its definition, even when the RDF is obtained in nonequilibrium conditions. Effective potentials have been used before in the context of cellular sheets [17], as they offer some intuition about the evolution of the system. However, similar to statistical potentials [26,27], the effective potential contains no more information than the RDF it has been derived from, and the link to quantities like the entropy (Sec. VII) is convenient yet equally well expressed in terms of the RDF.

The interpretation of the effective potential (3) in the cell-bead system corresponds to that of other areas in physics. In the present case, the effective potential quantifies attraction (accumulation) and repulsion (depletion) of beads by cells as a function of distance $r$. When the effective potential $U^{\text {eff }}(r, t, \tau)$ is equal to zero for all values of $r$, the system is (effectively) noninteracting. Thus, beads are uniformly distributed irrespective of the locations of cells, and there are no effective forces that disturb their spatial distribution. Negative values of the potential, $U^{\text {eff }}(r, t, \tau)<0$, imply an accumulation of beads, caused by an effective attraction of beads towards the given region in space. In contrast, positive values of the effective potential, $U^{\text {eff }}(r, t, \tau)>0$, are a signature of bead depletion, which can be caused by cells transporting beads away from that particular region in space.

When extracting the radial distribution function and effective potential from the experimental data, the observables are averaged over the entire population of single cells in the experiment (ensemble average), which is repeated for every experiment (details are given in methods Sec. A 4). 


\section{EXPERIMENTAL OBSERVATIONS}

In the absence of cells, beads were found to be uniformly distributed at the bottom of the well [Fig. 3(a)]. This distribution can be quantified by measuring the stationary bead-bead effective potential, Eq. (3). The measurements were performed 30 min after seeding the beads, allowing them to reach a stationary position at the bottom of the well, which implies that the effective potential is independent of both age and time delay, i.e., $U^{\text {eff }}(r, t, \tau)=U^{\text {eff }}(r)$. Thus, for this particular setup, we compute $U^{\text {eff }}(r)$ at a single time point and observe that the bead-bead effective potential shows small deviations from the null line, $U^{\text {eff }}(r)=0$. This result is expected for a uniform, independently random distribution of beads [Fig. 3(b)]. In this setting, bead-bead interactions occur only through volume exclusion, evidence of which is the positive deviation of the effective potential [Figs. 3(b)] for $r \approx 2.8 \mu \mathrm{m}$, corresponding to the diameter of a bead. In our experiments we consider low cell and bead densities, such that volume exclusion effects can be
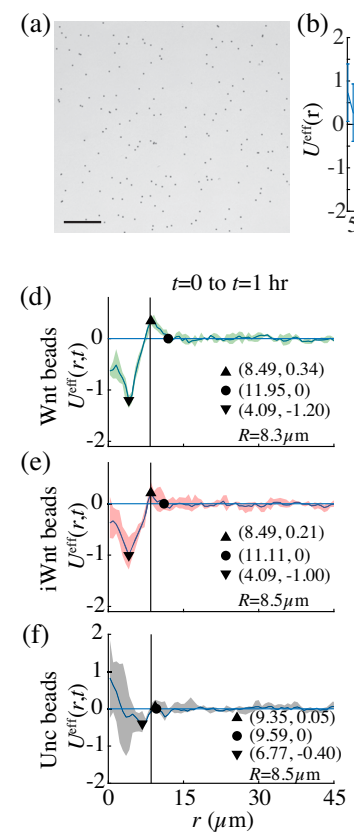

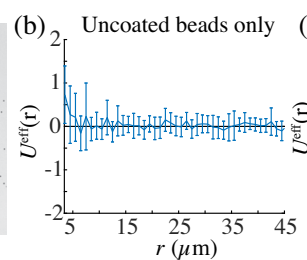

$t=4$ to $t=5 \mathrm{hr}$
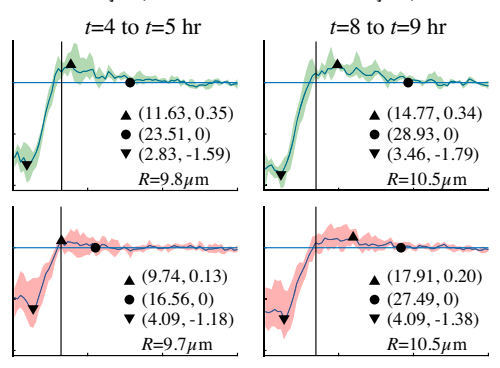

$R=10.5 \mu \mathrm{m}$
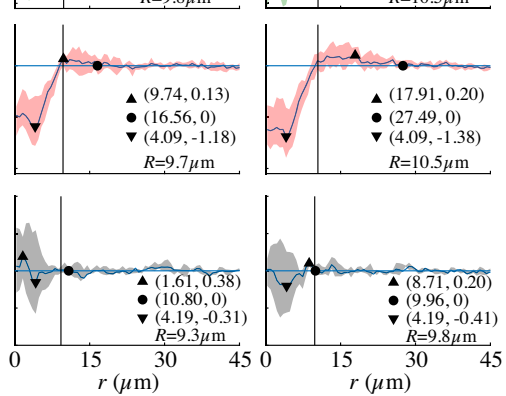

FIG. 3. (a) Snapshot of the experimental distribution of beads, and (b) the instantaneous effective potential $(\tau=0)$ for Unc beads and (c) Wnt beads, in the absence of cells. (d) Effective potential for Wnt beads, (e) iWnt beads, and (f) Unc beads in the presence of mESCs, taken over three different time windows (time increases from left to right, as indicated). The solid curve represents the mean across the repeats, while the shaded area represents the range of the data as obtained from the independent biological repeats. The reference line $U^{\text {eff }}(r, t)=0$ shows the effective potential of a uniform bead distribution. Indicated by (filled up-pointing triangle), (filled down-pointing triangle), and (filled circle) are the points of maximum depletion, accumulation, and the range, respectively (see main text). The vertical reference line indicates the mean cell radius $R$ at each time point. ignored. As expected, the protein-coated beads by themselves show the same behavior as Unc beads [Fig. 3(c)].

In general, the emergence of a nonzero effective potential is a consequence of the interaction between particles. In our system, interactions arise due to the presence of active mESCs, which act on the inanimate micron-sized beads. Beads move only by the effect of external forces, such as direct contact with cells, or by thermal fluctuations when they are in suspension in the liquid medium. Regardless of the type of bead coating considered (uncoated, Wnt, or iWnt), cells are always able to interact with beads. However, the properties of such interactions are greatly affected by the coating, which is evident even to the naked eye. Both the number of cells in contact with beads and the duration of the interaction is visibly higher in the case of coated beads compared to Unc beads, throughout the 12 hours of imaging. To quantify these observations, and to characterize the strength and properties of cell-bead interactions, we first measure the instantaneous effective potential $U^{\text {eff }}(r, t, \tau=0)$. Qualitatively, we consider the effective potential $U^{\mathrm{eff}}(r, t, \tau=0)$ at position $r$ as significant if the spread of measurements does not include $U^{\text {eff }}=0$.

When $\tau=0$, the location $r=0$ corresponds to the center of the cell; thus, the observable $U^{\text {eff }}(r, t, \tau=0)$ gives information on the preferred location of beads around the cell center. Measurements of the instantaneous effective potentials in the case of protein-coated beads show important differences in the interaction between mESCs and Wnt or iWnt beads, as seen in Figs. 3(d) and 3(e), respectively. Although the qualitative features of the effective potentials are similar between both conditions, their quantitative features differ. The interaction range, that is, the radial distance from the cell that is affected by depletion or accumulation $\left(U^{\text {eff }} \neq 0\right)$, quantifies the ability of cells to recruit beads from the environment [indicated by (filled circle) in Fig. 3], while the position of the minimum (maximum) of the effective potential indicates the most (least) probable position $r$ of a bead that interacts with a cell, indicated by filled down-pointing triangle (filled uppointing triangle).

The wider interaction range and deeper effective potential in Fig. 3(d), compared to that in Fig. 3(e), indicates a stronger interaction of cells with Wnt beads than with iWnt beads. When in contact with Wnt beads, mESCs appear to keep the beads closer to the center of the cell compared to cells in contact with iWnt beads, as seen when comparing the location of the potential minima (filled down-pointing triangle) in Figs. 3(d) and 3(e). These observations suggest that when in contact with a ligand, cells might control the ligand's spatial location, depending on its biochemical properties.

When cells are exposed to Unc beads, the interaction properties are drastically different compared to coated (Wnt or iWnt) beads. Unc beads have no ligand bound to their 
surface and thus provide no biochemical cue to the cells, acting merely as a physical entity in the environment. In this scenario, the instantaneous effective potential remains close to zero for the duration of the analysis [Figs. 3(f) and S3 in Supplemental Material [28] ]. Interactions with Unc beads are weak throughout the duration of the experiment, as measured by the low effective repulsion from the cell center $(r=0)$ at the initial stages and a shallow attraction at later time points. The wide dispersion in the data suggests that the interaction is quite volatile, possibly due to the lack of a proper biochemical coating preventing effective cellbead bonds. This case is in agreement with previous observations [29], which indicate that the presence of surface ligands favors the creation of durable chemical bonds between cells and external elements, such as artificial substrates, bioengineered matrices, and, in our case, micron-size beads.

To quantify the ability of cells to capture and retain beads, we calculate, in analogy to the theory of simple liquids, a time-dependent coordination number $c(t)$ [24],

$$
c(t)=2 \pi \rho_{b} \int_{0}^{R} \mathrm{~d} r \mathrm{e}^{-U^{\mathrm{eff}}(r, t, 0)} r,
$$

where $\mathrm{e}^{-U^{\text {eff }}(r, t, 0)}=\hat{g}(r, t, 0)$, and $R$ is the average radius of the cell. The coordination number, Eq. (4), is an approximated measure of the average number of beads around a cell at a given time $t$ [Fig. 4(a)]. The slope of the coordination number, indicated in Fig. 4(a), defines an accumulation rate, which quantifies how effective cells are in contacting and recruiting beads once they start probing the environment. A high rate suggests a deepening of the
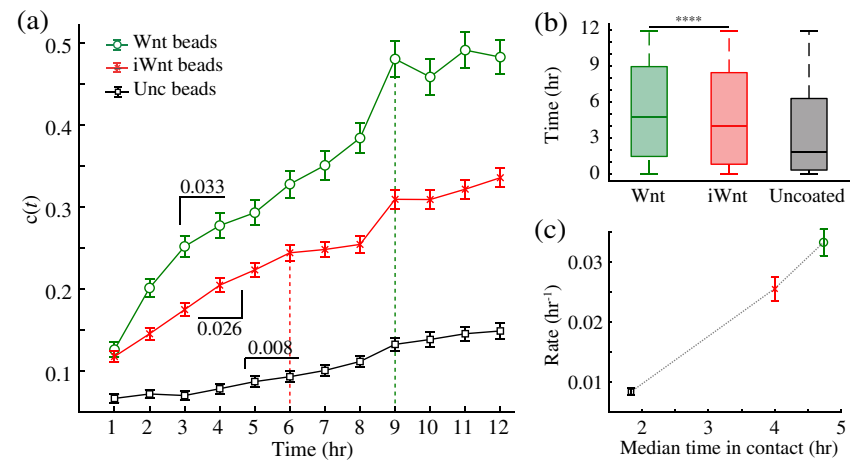

FIG. 4. (a) Coordination number $c(t)$, Eq. (4), as a function of time $t$ for the three bead types. Dashed lines indicate the time at which $c(t)$ starts plateauing for Wnt and iWnt beads. (b) Box plot of the effective time that beads remain in contact with cells, and (c) linear relationship between the accumulation rates in panel (a) and the median contact time in panel (b). In all panels, green, red, and black correspond to Wnt, iWnt, and Unc beads, respectively. Error bars in panels (a) and (c) show the 95\% confidence intervals. The statistical difference between the data distributions in panel (b) were calculated via a Kolmogorov-Smirnov test, where $^{* * * *}$ indicates a p-value less than 0.0001 . effective potential, an increase in bead accumulation, and the formation of new cell-bead pairs.

The accumulation rates [Fig. 4(a)] show a strong recruitment of signaling Wnt beads by mESCs, as the strength of the effective potential measured by the coordination number $c(t)$ increases at the fastest rate compared to all other beads. Moreover, the coordination number for iWnt beads begins to plateau at the 6-h mark, while Wnt-bead recruitment continues to increase for up to 9 hours. For Unc beads, the coordination number grows at a slow rate for the 12-h period [Fig. 4(a)]. These observations suggest that cells are able to retain Wnt beads for longer periods compared to iWnt and Unc beads, systematically increasing the overall number of cell-bead bonds in the system. This case is confirmed by measuring the amount of time that beads remain in contact with cells [Figs. 4(b) and 4(c)], which show significant differences between the three types of beads. The retention time is an effective quantity obtained from the difference between the first and last time points at which beads were considered to be in contact with a cell by the tracking algorithm (Sec. II and Fig. 2). Accumulation rates and retention times are linearly correlated [Fig. 4(c)] because longer retention times imply more stable cell-ligand bonds, which increase the overall accumulation of beads by cells. Here, durable and efficient cellbead contacts appear to rely strongly on the bead coating but also on the biological relevance of the coating, with cells retaining Wnt over iWnt beads for significantly longer periods. The observed dependence of the interaction strength on the bead coating is in line with related studies, where surface modifications of beads cause differential uptake of beads by cells [30,31].

In summary, mESCs actively interact with their surroundings-contacting, attracting, and repelling localized ligands. These interactions can be captured in the form of an effective potential, a statistical observable that gives information on the population-level organization of the system. The instantaneous effective potential $U^{\text {eff }}(r, t, \tau=0)$ reveals the range, strength, and aging of the interactions, yet it does not capture the effects of cell motility, which is a key feature of the cell dynamics. Beads are recruited and transported by direct contact with the cell membrane, which occurs due to cell movement and changes in cell shape. In order to observe the effect of cell dynamics on the effective potential, we now consider the effective potential with positive time delay, $\tau>0$.

\section{A. Time-delayed measurements}

The effect of a time delay $(\tau>0)$ on the measurements of the effective potential is a reduction of the strength of the attraction, as seen in Fig. 5. This flattening of the effective potential is caused by cell motility. For a given time delay $\tau$, the distribution of beads at time $t+\tau$ is measured with respect to the positions of cells at time $t$. Any displacement of the cell between $t$ and $t+\tau$ affects the reference point 

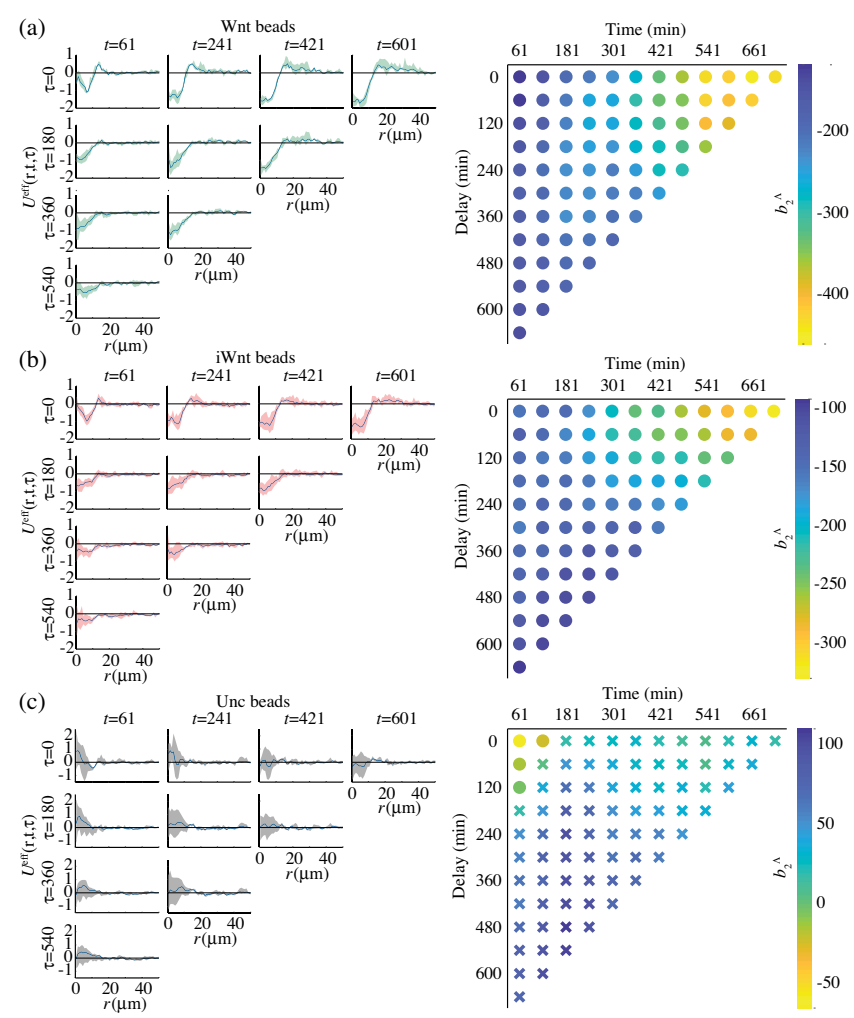

FIG. 5. Experimental effective potential as a function of time $t$ and delay $\tau$ (left panel) and $(t, \tau)$-phase diagram (right panel), for (a) Wnt beads, (b) iWnt beads, and (c) Unc beads, in the presence of cells. Markers indicate attractive (circles, negative $b_{2}^{\Lambda}$ ) and repulsive (crosses, positive $b_{2}^{\Lambda}$ ) regions in the phase diagrams, as measured by Eq. (5) for $\Lambda=2 R$ (color bar), where $R$ is the cell radius.

from which the measurement of the radial distribution function Eq. (2) and thus the effective potential Eq. (3) are taken. For large values of $\tau$, the effective potential can become flat for all values of $r$, resembling an effectively noninteracting system (Fig. 5, left column). To characterize this and other changes of the effective potential as a function of both age $t$ and time delay $\tau$, we construct a phase diagram in the $(t, \tau)$ plane (Fig. 5, right column). Each point in the phase diagram is obtained by integrating the Mayer $f$-function $f(r, t, \tau)=\mathrm{e}^{-U^{\text {eff }}(r, t, \tau)}-1[24,32]$,

$$
b_{2}^{\Lambda}(t, \tau)=-\pi \int_{0}^{\Lambda} \mathrm{d} r f(r, t, \tau)
$$

In equilibrium systems and in the limit $\Lambda \rightarrow \infty$, Eq. (5) corresponds to the second virial coefficient [32], which accounts for the first-order corrections to the pressure in low-density systems. For large values of $\Lambda$, the behavior in the phase diagram changes considerably as contributions from large distances, where the noise in the data is larger, dominate the value of $b_{2}^{\Lambda}$ (Fig. S8 in Ref. [28]). In our case, we are interested in the behavior of the effective potential around the cell; thus, we consider a finite upper cutoff $\Lambda=2 R$, where $R$ is the cell radius. Negative values of $b_{2}^{\Lambda}(t, \tau)$ indicate a deep, attractive effective potential, while positive values indicate effective repulsion around the cell center [24].

As expected, at fixed time $t$, the overall effect of increasing the delay $\tau$ is to reduce the effect of attractive interactions [Figs. 5(a) and 5(b), right column], as the effective potential approaches zero. On the other hand, for a fixed delay $\tau$, the overall effect of aging, i.e., of increasing $t$, is to increase the strength of the effective potential. However, for Unc beads [Fig. 5(c)], the phase diagram appears to transition from attractive at short times and delays to purely repulsive at longer times and delays. Here, the phase diagram appears inverted with respect to that of coated beads, suggesting that a different interaction mechanism might be taking place compared to Wnt and iWnt beads. We already noted that interactions are reduced in the case of Unc beads (see Fig. 3); however, this does not explain the inversion observed in the phase diagram, where the effective potential appears to become stronger and more repulsive with increasing time $t$ or delay $\tau$.

To obtain a comprehensive understanding of the interplay between the cell dynamics, age, time delay, and effective potential, we introduce, in the following, a simple theoretical model for the cell-ligand interactions. Together with numerical simulations, this model allows us to identify the causes of the aging of the effective potential. Moreover, it explains the differences between the phase diagrams in Fig. 5.

\section{THEORETICAL AND NUMERICAL ANALYSIS}

Our model of cell-bead interactions considers two species of particles in a continuous, finite, two-dimensional space. First, immobile point particles, corresponding to beads [black particles in Fig. 1(b)], were distributed randomly on a unit square with periodic boundary conditions (a torus) so that their number density was $\rho_{b}=1000$. Second, a mobile, single cell was placed at the origin of the unit square. This cell corresponds to an active mESC, modeled as a diffusive disk of radius $r_{0}$ [black circle in Fig. 1(b)]. At each time step, the cell moves a small fixed distance $\delta<r_{0}$ in a random direction on the torus, which amounts to a diffusion constant $D=\delta^{2} / 4$ (see methods Sec. A 1 for details). A bead is captured by a cell if it lies within the cell's disk after a time step. Once caught, beads [blue particles in Fig. 1(b)] are distributed inside the corresponding cell according to a normal distribution with standard deviation $\sigma=r_{0} / 2$, centered on the cell's center and truncated at $r_{0}$. The choice of distribution does not affect the quantitative results presented below; it does, however, modify the precise shape of the effective potential. Initially, we do not allow for the release of beads captured by cells, such that bead capturing is an irreversible process and is the only explicit interaction between beads and cells. 

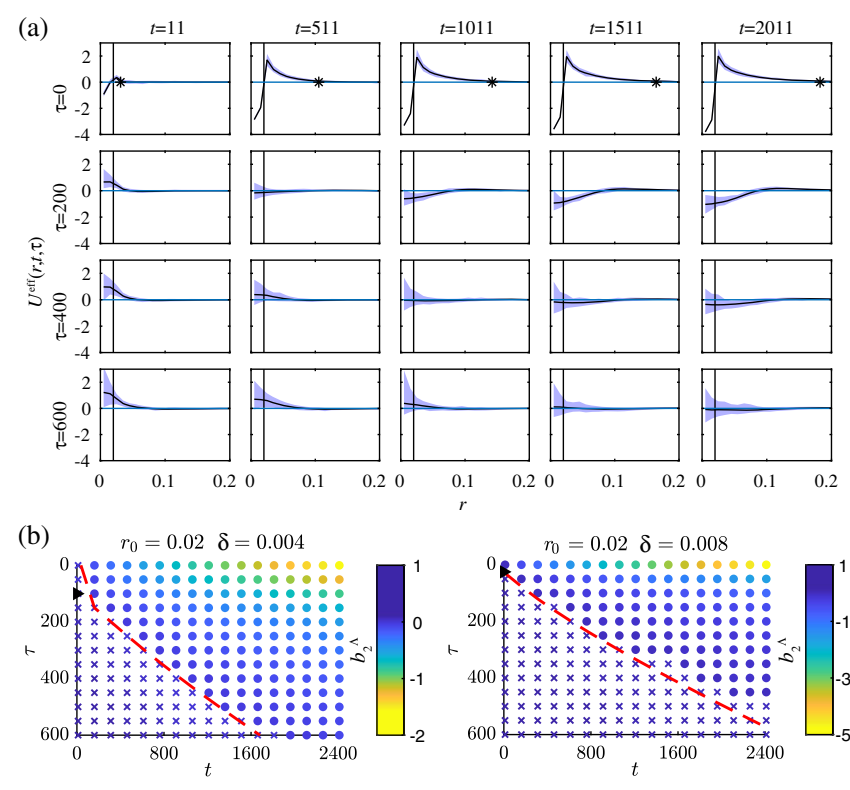

$r_{0}=0.02 \quad \delta=0.015$
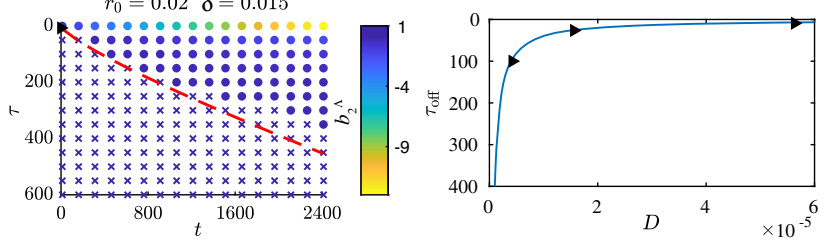

FIG. 6. (a) Effective potential as a function of age $t$ and delay $\tau$ obtained from numerical simulations of the model described at the beginning of Sec. V, indicating the mean (solid curve) and range (shaded) from $10^{4}$ realizations, for step size $\delta=0.004$ and cell radius $r_{0}=0.02$ (vertical line). The symbol $(*)$ indicates the interaction range $r_{e}(t)=\sqrt{A / \pi}$. (b) The $(t, \tau)$-phase diagram for increasing values of step length $\delta$. Markers indicate attractive (circles, negative $b_{2}^{\Lambda}$ ) and repulsive (crosses, positive $b_{2}^{\Lambda}$ ) regions in the phase diagrams, as measured by Eq. (5) for $\Lambda=2 R$ (color bar), where $R$ is the cell radius. The symbol (filled left-pointing triangle) indicates the predicted delay offset, Eq. (9); the dashed line corresponds to the theoretical transition delay $\tau^{*}$, obtained from Eq. (11). The potential is effectively repulsive below the line (longer delays, crosses) and effectively attractive above (shorter delays, circles). The last panel shows the delay offset as a function of diffusivity, $D=\delta^{2} / 4$, obtained from Eq. (9); the triangles indicate the delay marked on the phase diagrams.

In the model described above, the master equation for the probability $P(n, t)$ that a cell has captured $n$ beads up to time $t$ may be written as

$$
\frac{d P(n, t)}{d t}=\rho_{b} \frac{d A}{d t}[P(n-1, t)-P(n, t)]
$$

where $\rho_{b}$ is the number density of beads, and $A=$ $A\left(D, r_{0}, t\right)$ corresponds to the area covered by the diffusive disc, representing the cell, of radius $r_{0}$ and diffusion constant $D$ up to time $t$, sometimes referred to as the area of the Wiener sausage $[33,34]$. To first order, the area explored by the cell grows like

$$
A\left(D, r_{0}, t\right) \approx \frac{4 \pi D t}{\log \left(\beta \frac{D t}{r_{0}^{2}}\right)}
$$

in the long time limit, where $t \gg r_{0}^{2} / D$. Here, $\beta=$ $4 \exp (-2 \gamma)$, and $\gamma \approx 0.577$ is the Euler-Mascheroni constant [33]. As the upper-critical dimension of a random walk is two, we may assume that $\rho_{b}$ is constant in space and time [35]. Under this assumption, Eq. (6) can be solved explicitly, so

$$
P(n, t)=\frac{\left[\rho_{b} A\left(D, r_{0}, t\right)\right]^{n}}{n !} e^{-\rho_{b} A\left(D, r_{0}, t\right)} .
$$

The expected number $\langle n(t)\rangle$ of beads captured by a cell as a function of time is then proportional to the area explored by the cell up to time $t,\langle n(t)\rangle=\rho_{b} A$ [green area in Fig. 1(b)]. The excess number of beads within the cell radius $n_{\mathrm{ex}}(t)=$ $\langle n(t)\rangle-\pi r_{0}^{2} \rho_{b}$ determines the level of accumulation of beads, i.e., the depth of the instantaneous effective potential $\left[U^{\text {eff }}(r, t, \tau=0)<0\right]$. At the same time, the accumulation of beads by the cell is proportional to the depletion of beads in the region surrounding it [see Fig. 6(a), first row]. Thus, the excess number of beads $n_{\mathrm{ex}}(t)$ can be regarded as a proxy for the strength of the effective potential. The irreversible nature of the capturing process implies that $n_{\mathrm{ex}}(t)$ is a monotonically increasing function; thus, the strength of the effective potential is bound to increase with time in the present setting.

The effective radius of the area explored by a diffusive cell, $r_{\mathrm{e}}(t)=\sqrt{A\left(D, r_{0}, t\right) / \pi}$ [indicated by $*$ in Fig. 6(a)] corresponds approximately to the interaction range of the effective potential, as it indicates the extent to which the environment is affected by the action of a cell. The combined evolution of $n_{\mathrm{ex}}(t)$ and $r_{\mathrm{e}}(t)$ explains the experimental observation for mESCs with Wnt and iWnt beads - that the effective potentials get stronger and longerranged with time, as a result of capturing and transport of beads by cells.

Considering positive time delay $(\tau>0)$ in the measurements causes important changes of the effective potential. For increasing values of $\tau$, the accumulation region becomes shallower, projecting further than the radius of the cell [Fig. 6(a)]. This result is a consequence of the displacement of the cell (and the captured beads) between time $t$, at which the positions of cells are considered, and time $t+\tau$, at which the bead distribution is measured, in order to compute the RDF, Eq. (2). The root-mean-square displacement $\ell$, of the cell between these two times, $t$ and $t+\tau$, is related to the diffusion constant by $\ell(\tau)=\sqrt{4 D \tau}$.

Interestingly, as the delay $\tau$ is increased further, we find a transition in the phase diagram, where the attractive range around $r=0$ disappears, giving rise to a purely repulsive potential [Fig. 6(a) for $\tau>0$ ]. This transition happens despite the attractive nature of the capturing mechanism, 
the only explicit interaction in our model. To understand how and when this transition occurs, we look at the $(t, \tau)$ phase diagram for the numerical results with positive delay, $\tau>0$.

\section{A. Effective repulsion}

The effective repulsion is characterized by the effective potential being positive at the origin, $U^{\text {eff }}(r=0, t, \tau)>0$, and decaying towards zero with increasing distance, $r>0$. As the only characteristic timescale in the system is given by the diffusive timescale of cells, we expect that the transition between the two regimes, attractive and purely repulsive in the $t-\tau$ plane [dotted line in Fig. 6(b), is controlled by the diffusivity $D$, defined above. In our numerical simulations of the model introduced at the beginning of this section, we observe that there is a minimum time delay $\tau$ that has to pass for the transition to be observed, which depends on the cell's step length. This delay offset $\tau_{\text {off }}$ marks the beginning of the transition of the effective potential from attractive [dots in Fig. 6(b)] to repulsive [crosses in Fig. 6(b)]. The delay offset $\tau_{\text {off }}$ is related to the time it takes for a cell to displace itself and the beads attached to it by a distance of the order of the cell size, i.e.,

$$
\tau_{\text {off }} \approx \frac{r_{0}^{2}}{D}
$$

as shown in Fig. 6(b) (last panel). This net transport of beads causes the effective potential $U^{\text {eff }}\left(r, t, \tau>\tau_{\text {off }}\right)$ to be positive (repulsive), on average, around $r=0$, for long delays $\tau>\tau_{\text {off }}$.

The offset $\tau_{\text {off }}$ explains why, in the experiments for Wnt and iWnt beads, we do not observe the potential being only repulsive. The values for the diffusion constant of the cells in the experiments vary slightly between conditions, with $D \approx 0.21 \mu^{2} /$ min for cells in the presence of uncoated and Wnt beads, and $D \approx 0.23 \mu^{2} / \mathrm{min}$ for cells in the presence of iWnt beads (methods Sec. A $1 \mathrm{a}$ ). Using the average radius of the mESCs, $r_{\mathrm{sc}} \sim 10 \mu \mathrm{m}$, we obtain from Eq. (9) estimates for the expected delay offset $\tau_{\text {off }}$ in the experiments. These estimates are $\tau_{\text {off }} \approx 470$ min for Wnt beads and $\tau_{\text {off }} \approx 430 \mathrm{~min}$ for iWnt beads. These predictions for the onset of the transition of the effective potential turning repulsive are compatible with the experimental observations. For the earliest experimental time window $(t=61 \mathrm{~min})$, we observe that the smallest delays for which the accumulation region around $r=0$ crosses the reference $U^{\text {eff }}=0$ towards positive values are $\tau=\tau_{\text {off }} \sim$ $420 \mathrm{~min}$ for Wnt beads (see Ref. [28], Fig. S1, first column) and $\tau=\tau_{\text {off }} \sim 360$ min for iWnt beads (see Ref. [28], Fig. S2, first column).

Numerically, we can study regimes that are inaccessible in our experimental setup. For $t \sim 0$, once the delay offset has been exceeded, i.e., $\tau>\tau_{\text {off }}$, the potential transitions to effective repulsion [dashed lines, Fig. 6(b)]. For later times $t>0$, the transition occurs after longer delays, taking place when the displacement $\ell(\tau)=\sqrt{4 D \tau}$ of the cell between times $t$ and $t+\tau$ is of the order of the effective radius $\sqrt{A / \pi}$ of the area $A\left(D, r_{0}, t+\tau\right)$ explored by the cell until time $t+\tau$,

$$
4 \pi D \tau^{*}=A\left(D, r_{0}, t+\tau^{*}\right),
$$

so that any bead within the cell's effective range of interactions at that time, $\sqrt{A / \pi}$, has been displaced over the course of time $\tau$ by about that distance. Therefore, the transition occurs at

$$
\tau^{*}=\frac{\exp \left(1+\frac{t}{\tau^{*}}\right)}{\left(1+\frac{t}{\tau^{*}}\right)} \frac{r_{0}^{2}}{\beta D}
$$

using Eq. (7), which is valid for $t \gg r_{0}^{2} / D$. At larger delays, the apparent displacement of beads away from the effective origin $r=0$ exceeds the effective range of interactions, $\sqrt{A / \pi}$, so the potential appears to be repulsive. Numerically solving the transcendental equation (11) for $\tau^{*}$ produces the dashed transition lines shown in Fig. 6(b). Below this line (longer delays), the potential typically appears to be repulsive, and above this line (shorter delays), it typically appears to be attractive.

The delayed effective potential serves as a tool to quantify the transport of ligands or beads by cells. In numerical simulations, motility is the only mechanism available for cells to encounter beads. However, in the experimental conditions, we have observed that mESCs constantly change their shape, as they elongate and increase their interaction range without net displacement. Moreover, mESCs do not necessarily capture every single bead they encounter, disregarding some of the beads they come in contact with. Furthermore, there can be transient interactions; a cell can recruit a bead that then spontaneously detaches from the cell membrane. In the next section, we study the effect on the effective potential of both of these mechanisms independently by incorporating a disregarding probability and a Poissonian release of beads to our model. We consider two possible release scenarios: one in which beads are recruited by the cells and subsequently released in a random direction without interacting with them ever again, and a second one where cells can repeatedly interact with beads. In the latter, beads are effectively pushed by the cells as they release the beads in the direction they were encountered.

\section{DISREGARDING AND RELEASING BEADS}

The effect of mESCs disregarding a fraction of beads encountered can be incorporated into the master equation (6) by introducing a disregarding probability $\chi$. The density of beads that a cell can interact with is then 
effectively reduced to $\rho_{\text {eff }}=(1-\chi) \rho_{b}$, with $\chi \in[0,1]$. This reduction represents the affinity of the cell-ligand interactions. The master equation, in this case, reads

$$
\frac{d P_{\chi}(n, t)}{d t}=\rho_{\text {eff }} \frac{d A}{d t}\left[P_{\chi}(n-1, t)-P_{\chi}(n, t)\right] .
$$

As $\chi$ approaches unity, an increasingly large proportion of beads is disregarded, thus increasing the mean free path of the cell. By introducing rescaled time $t^{\prime}=(1-\chi) t$ and cell radius $r_{0}^{\prime}=\sqrt{1-\chi} r_{0}$ in Eq. (12), where $A$ is given by Eq. (7), we recover the form of Eq. (6). This rescaling shows that larger values of $\chi$ imply a slower evolution of the effective potential (see Fig. S5); however, its qualitative properties, such as the potential's overall shape, and the existence of the transition between attractive and repulsive regimes, are not affected by the introduction of the disregarding probability [see Fig. 7(a)]. Disregarding beads could be responsible for the differences observed in the properties of the effective potentials between Wnt beads [Fig. 5(a)] and iWnt beads [Fig. 5(b)]. As described above, the effective potentials and phase diagrams evolve in a qualitatively similar manner but with the dynamics sped up for the signaling Wnt beads. This change of timescale
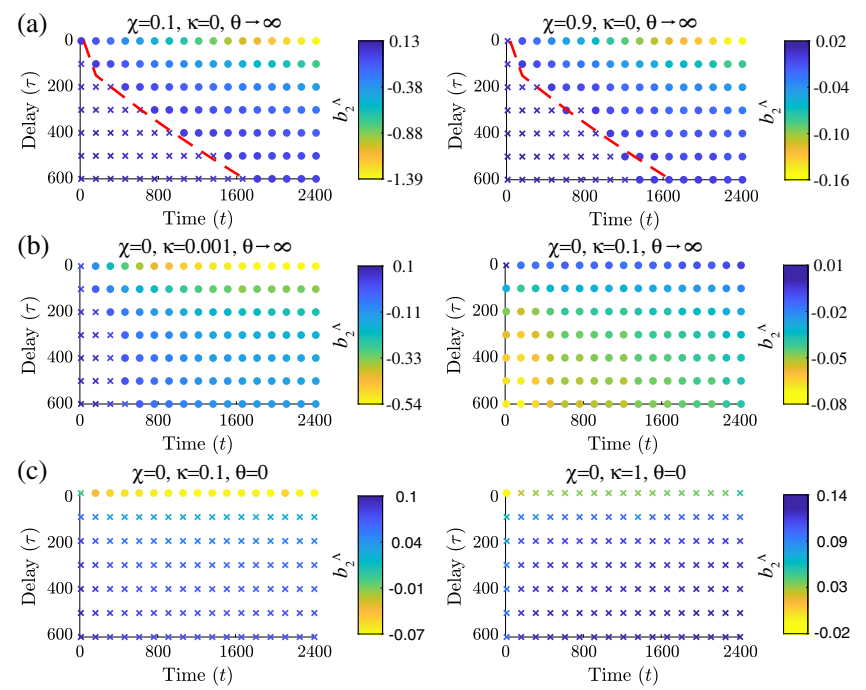

FIG. 7. ( $t, \tau)$-phase diagram of the model with (a) positive disregarding probability $\chi$, (b) positive release probability $\kappa$, and (c) positive release probability $\kappa$ with refractory time $\theta=0$, for increasing values (left to right). Simulations consider a single cell of radius $r_{0}=0.02$ performing a random walk with step length $\delta=0.004$, in a periodic system of linear size $L=1$ and bead density $\rho_{b}=10^{3}$. The dashed lines in panel (a) represent the predicted transition from attractive to repulsive according to Eq. (11). Markers indicate attractive (circles, negative $b_{2}^{\Lambda}$ ) and repulsive (crosses, positive $b_{2}^{\Lambda}$ ) regions in the phase diagrams, as measured by Eq. (5) for $\Lambda=2 R$ (color bar), where $R$ is the cell radius. In panel (c), the release of beads occurs in the same direction in which they were encountered by the cell, effectively pushing beads away. suggests that cells have a lower affinity for nonsignaling iWnt beads. In other words, cells are less likely to recruit iWnt beads.

As suggested by the measurements of the total time that beads remain in contact with the cell [Fig. 4(c)], cell-bead bonds are reversible, so beads occasionally detach from the cell membrane, getting released to the environment. To account for this process, we introduce to our model a Poissonian release rate $\nu \in[0, \infty)$. In the numerics, we consider a time step $\Delta t=1$. Hence, in every iteration, the release probability $\kappa$ that an attached bead will be released by the cell is given by $\kappa=1-\mathrm{e}^{-\nu \Delta t}$. At this stage, released beads are considered to simply decouple from the movement of the cell, and their position is held fixed during further evolution of the process so that the cell disregards released beads without ever recapturing them. Without this simplification, any released bead would be subject to instantaneous re-recruitment (this corresponds to taking the limit of $\theta \rightarrow \infty$ of the refractory time introduced below). A diffusing cell will therefore leave a trail of released beads along its path. This simple release mechanism naturally lends itself to a description in terms of a master equation. Given the probability $P(n, t)$ of observing $n$ beads in contact with a cell at time $t$, the rate of decay of that state into a state with $n-1$ beads is $\nu n P(n, t)$. Taking this rate into account, the master equation for the probability $P_{\nu}(n, t)$ reads

$$
\begin{aligned}
\frac{d P_{\nu}(n, t)}{d t}= & \rho_{b} \frac{d A(t)}{d t}\left[P_{\nu}(n-1, t)-P_{\nu}(n, t)\right] \\
& +\nu\left[(n+1) P_{\nu}(n+1, t)-n P_{\nu}(n, t)\right] .
\end{aligned}
$$

This equation can be solved via a generating function approach (see methods Sec. A 5 for details) to find the explicit solution

$$
P_{\nu}(n, t)=\frac{\alpha^{n}(A, \nu ; t)}{n !} e^{-\alpha(A, \nu ; t)},
$$

where $\alpha(A, \nu ; t)$ is a function of the area $A=A\left(D, r_{0}, t\right)$ explored, Eq. (7), the release rate $\nu$, and time $t$,

$$
\alpha(A, \nu, t)=\rho_{b} \int_{0}^{t} \mathrm{~d} s \frac{d A(s)}{d t} e^{-\nu(t-s)} .
$$

Given the structure of the solution, Eq. (14), $\alpha$ is in fact the expected number of beads attached to a cell, $\langle n(t)\rangle=\alpha(A, \nu, t)$, in the presence of Poissonian decay by detachment. For $\nu=0$, Eq. (8) is recovered, where $\langle n(t)\rangle=\rho_{b} A(t)$. For $\nu>0, \alpha$ is a concave function (see Fig. S4); hence, there is a time $t=t^{*}$ for which the Poissonian release overcomes the capturing of beads by the cell exploring its environment. Before $t=t^{*}$, the average number of beads captured, $\langle n(t)\rangle$, and consequently the accumulation strength of the effective potential, 
increases with time. At $t=t^{*}$, the parameter $\alpha(A, \nu ; t)$ and thus $\langle n(t)\rangle$ reach their maximum values. For $t>t^{*}$, the number of captured beads decreases, and the effective potential weakens, asymptotically recovering the noninteracting behavior $U^{\text {eff }}=0$.

The impact of the release mechanism on the effective potential (Fig. S6) and phase diagram [Fig. 7(b)] becomes most evident at positive delay, $\tau>0$. For increasing values of the release probability, the transition between attractive and repulsive phases shifts towards earlier times $t$ [Fig. 7(b)]. For large values of $\kappa$, the phase diagram is (almost) purely attractive, weakening with age $t$ and strengthening with delay $\tau$. This case is in contrast to the observations for $\chi>0$ and $\kappa=0$ [Fig. 7(a)].

The phase diagram for large release probability $\kappa=0.1$ in Fig. 7(b) (right) appears to be inverted compared to the phase diagram for small release probability $\kappa=0.001$ to its left, as the locations of strongest and weakest attraction have swapped position in the $t-\tau$ plane. The release mechanism helps explain the difference in the phase diagram observed between Wnt beads, on the one hand, and Unc beads, on the other; however, the almost pure attraction for $\kappa=0.1$ observed in the model is in sharp contrast to the almost pure repulsion seen for Unc beads in the experiments, Fig. 5(c). A key observation in the experiments is that $\mathrm{mESCs}$ interact with Unc beads, yet are unable to recruit them, as quantified by the effective potential [Fig. 5(c)]. In the experiments, Unc beads encountered by a cell tend to remain on its periphery. As it moves, the cell effectively pushes beads in the direction of its movement. As the upper critical dimension of Brownian motion is two, its motion requires each cell to interact repeatedly with every bead it encounters. To incorporate this process into the numerics, and to take into account possible memory effects, we introduce a refractory time $\theta$, which has to lapse after releasing a bead before the cell can recapture that same bead. The release mechanism as introduced above, whereby released beads remain immobile irrespective of the movement of a cell, thus amounts to an infinite refractory time. In the limiting case of diverging release rate $\nu(\kappa=1)$ and vanishing refractory time $\theta$, beads are continuously pushed away by the cell. For finite values of $\nu(\kappa<1)$, beads can be transported by the cell and released at a later time point. We implement the release in this pushing dynamics by placing the bead at the point of the cell periphery where it was first encountered. This method accounts for the experimental observation that the bead is not transported on top of the bulk cell membrane but near its boundary where it is in contact with the substrate.

The phase diagram for the pushing mechanism [Figs. 7(c) and S7], i.e., $\theta=0$, such that the cell can reinteract with beads in every iteration, shows that with increasing release rates, the attractive region disappears. For $\kappa \approx 1$, the phase diagram transitions from being repulsive for very short times and delays, to purely repulsive for longer times and delays, a feature previously observed in the experiments of mESCs with Unc beads [Fig. 5(c)]. In both the simulations and experiments, the effective interactions become stronger, i.e., more repulsive with both age $t$ and delay $\tau$, in contrast to what was observed for coated beads [Figs. 5(a) and 5(b)].

The results presented in this section, together with the effective retention times [Fig. 4(c)[, suggest that the cellligand interactions are strongly affected by the details of the disregarding and releasing mechanisms. The absence of coating seems to impede the cells' capacity for actively recruiting beads and thus organize their microenvironment.

\section{CONFIGURATIONAL ENTROPY}

The ability of cells to interact with and organize their environment is evidenced by the emergence of the effective potential of interactions. As observed experimentally, and demonstrated through numerical simulations, different cellligand interaction mechanisms can contribute to a higher or lower organization in the spatial distribution of beads. Changes in the distribution of beads affect the excess configurational entropy per cell $s_{c}$, a quantitative measure of the level of spatial order in the system. In our setup, where the area fractions covered by both cells and beads are low, the configurational entropy per cell $s_{c}$ can be written perturbatively, to first order, in terms of the radial distribution function [36],

$$
s_{c}=\pi \rho_{c} \int \mathrm{d} r\left[\hat{g} U^{\mathrm{eff}}+(\hat{g}-1)\right] r,
$$

where $U^{\text {eff }}=-\ln (\hat{g})$ and $\rho_{c}$ is the cell density. This expression is generally valid for dilute suspensions in equilibrium, where $\hat{g}$ is independent of time and the states of the system follow a Boltzmann distribution. In the cellbead system, where the correlations are time dependent, and with many thermodynamic properties undefined, we use Eq. (16) as an effective measure of the configurational entropy at each time point. With this definition, the rate of change of the configurational entropy, referred to as the entropy rate hereafter, reads $\dot{s}_{c}(t)=\pi \rho_{c} \int \mathrm{d} r \sigma(r, t) r$, with density

$$
\sigma(r, t)=\dot{\hat{g}}(r, t, \tau=0) U^{\mathrm{eff}}(r, t, \tau=0) .
$$

This entropy rate is the slope of the configurational entropy as a function of time (Fig. 8). The more negative, the more the cells contribute to organizing their environment. For a noninteracting homogeneous system, the radial distribution function $\hat{g}$ is unity and the effective potential $U^{\text {eff }}$ vanishes, so the entropy rate $\dot{s}_{c}$ vanishes identically. The configurational entropy decreases as the system becomes more organized. This entropy reduction carries an energy cost, which is provided by the active cells that transport beads and keep the system out of equilibrium. 

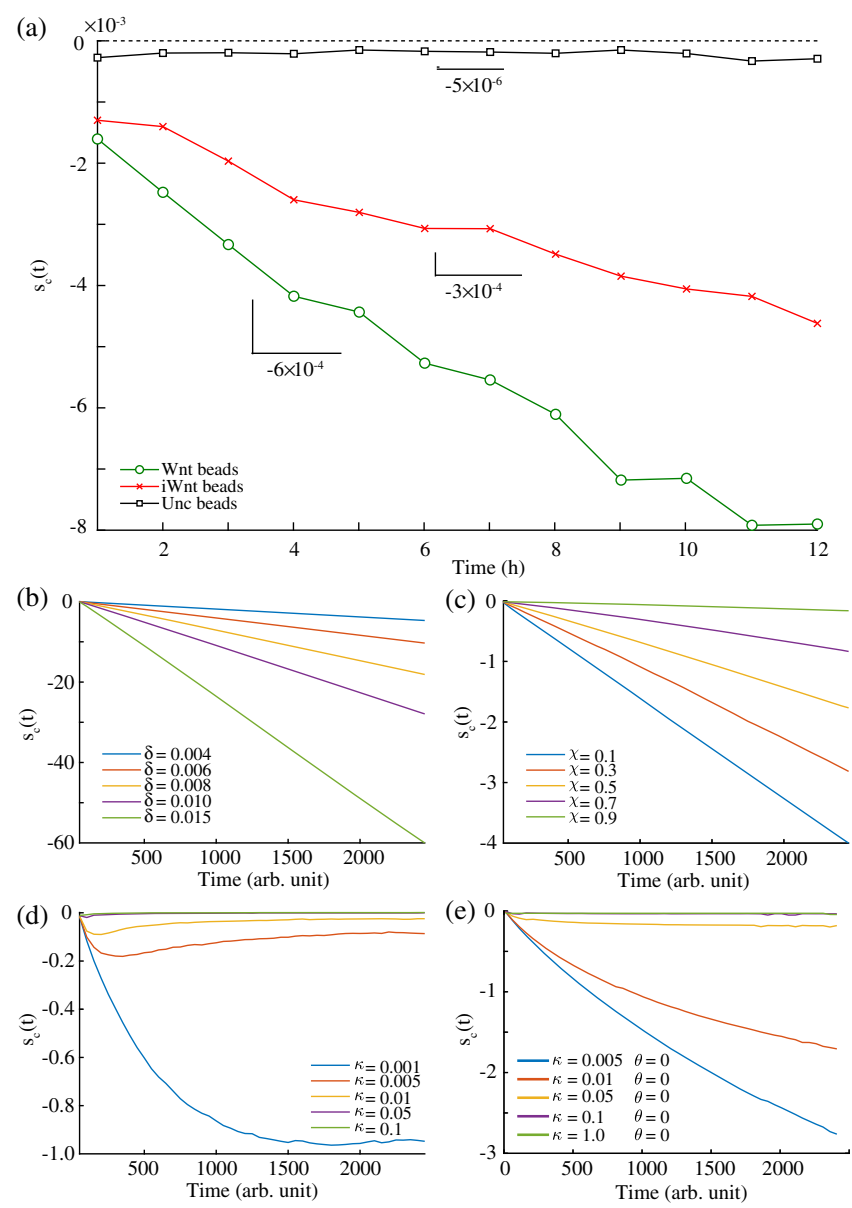

FIG. 8. Effective configurational entropy as a function of time for (a) the experimental conditions, (b) the numerical simulations of the model described at the beginning of Sec. V for different step lengths $\delta$, (c) the model with positive disregarding probability $\chi$ but no release mechanism, Eq. (12), (d) the model with positive release probability $\kappa$ but infinite refractory time, Eq. (13), and (e) the model with positive release probability $\kappa$ and refractory time $\theta=0$. The dashed line in panel (a) corresponds to the reference line $s_{c}=0$. Simulations in panels (b)-(e) consider a single cell of radius $r_{0}=0.02$, in a periodic system of linear size $L=1$, and bead density $\rho_{b}=10^{3}$. The step length for the simulations in panels (c)-(e) is set to $\delta=0.004$. In panel (e), the release of beads occurs in the same direction in which they were encountered by the cell, effectively pushing beads away.

As expected from the observation of attractive effective potentials for Wnt and iWnt beads [Figs. 3(d) and Fig. 3(e)], the dynamics of these systems tends to decrease the configurational entropy $s_{c}$, lowering the total entropy of the bead distribution. In both cases, the configurational entropy decreases linearly in time [Fig. 8(a)]; thus, the entropy rate is roughly constant. The entropy for Wnt beads decreases at a rate almost twice that of nonsignaling iWnt beads, suggesting that the biochemical signaling of Wnt ligands contributes to a faster organization of the system. The configurational entropy of Unc beads, on the other hand, shows no significant change in time. It remains near the noninteracting reference $s_{c}=0$ for the 12-h period of observation, a consequence of the lack of recruitment and organization of Unc beads by mESCs.

As argued in the previous section, the behavior of mESCs with Wnt beads is well described by the simplest model (beginning of Sec. V) for which we observe a linear decrease of configurational entropy over time [Fig. 8(b)]. The rate depends on the step length and thus the diffusion constant of the cells. However, the diffusivity does not vary significantly between experiments (see Sec. VA) and therefore does not suffice to explain the higher entropy rate observed when cells interact with Wnt beads compared to nonsignaling iWnt beads. By introducing the disregarding probability $\chi$ [Fig. 8(c)], we were able to replicate the experimental observations, where for a fixed diffusion constant and positive probability $\chi>0$, the magnitude of the entropy density rate is reduced compared to $\chi=0$, but it remains constant in time.

When taking into account the release of beads with probability $\kappa$ and subsequent disregarding, $\theta \rightarrow \infty$ [Fig. 8(d)], the entropy reaches a minimum when the mean number of beads captured is maximum (see Fig. S4). After this point, the entropy generation becomes slightly positive before leveling off so that $s_{c}(t)$ plateaus, closer to 0 with increasing $\kappa$. When considering the pushing mechanism (large $\kappa$, small refractory time $\theta$ ) described at the end of the previous section, the entropy also reaches a plateau [Fig. 8(e)]. For high release probability $\kappa$, the configurational entropy
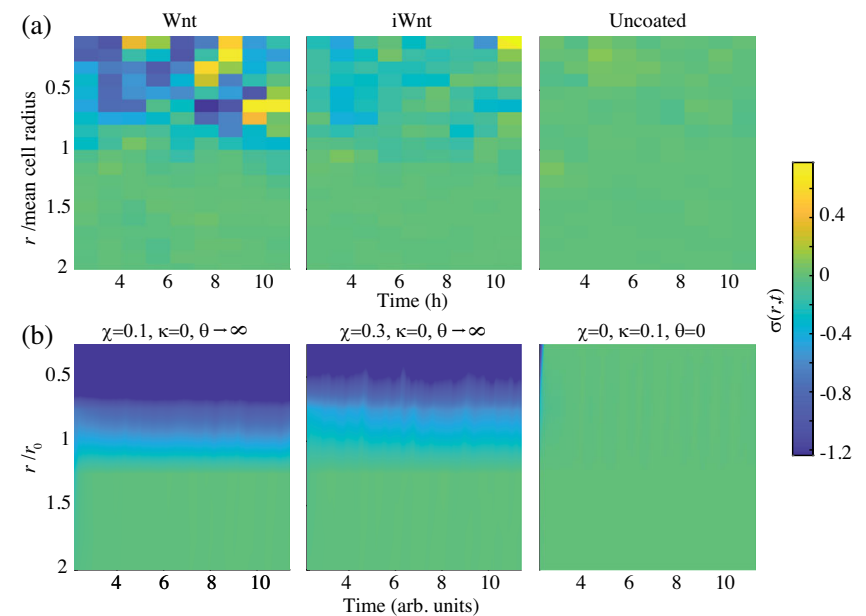

FIG. 9. Entropy density rate $\sigma(r, t)$, Eq. (17), as a function of distance $r$, in units of the cell radius, and time $t$ for (a) the experimental conditions and (b) the numerical simulations of the model with irreversible cell-bead binding, Eq. (6) (left panel), the model with positive disregarding probability $\chi$, Eq. (12) (center panel), and the model with positive release probability $\kappa$ and refractory time $\theta=0$ (right panel). Simulations in (b) consider a single cell of radius $r_{0}=0.02$, in a periodic system of linear size $L=1$, and bead density $\rho_{b}=10^{3}$. The step length for the simulations is set to $\delta=0.004$. The color scale is the same for all plots. 
remains constant, close to zero for the whole observation time. This behavior was also observed for Unc beads [Fig. 8(a)], where the entropy remains essentially constant for the 12-h period.

The entropy density rate $\sigma(r, t)$ shows that, in the case of signaling Wnt beads [Fig. 9(a), left panel], the largest contribution to negative entropy generation occurs around $r=0$, close to the cell center. This case is consistent with numerical simulations] Fig. 9(b)], where, in addition, $\sigma(r, t)$ remains approximately constant over time $t$. As expected from their weaker effective potentials compared to Wnt beads (see Ref. [28], Figs. S1-S3), the density $\sigma$ is significantly reduced for nonsignaling iWnt beads and essentially vanishes for Unc beads [Fig. 9(a)].

\section{CONCLUSIONS AND OUTLOOK}

In this work, we made use of a simplified experimental setup, in which active mESCs interact with ligands that are immobilized on the surface of micron-sized beads. We performed time-lapse imaging at high spatiotemporal resolution and followed the dynamics of single cells interacting with homogeneously distributed beads. By introducing the age- and time-dependent effective potentials, we characterized the spatiotemporal dynamics of cell-ligand interactions as an aging system of two interacting species of particles.

Effective potentials have been extensively used in the stochastic and hydrodynamic modeling of active matter $[12,13]$. However, until now, these potentials had only been measured experimentally in stationary, confluent cell sheets [17]. Our results are the first measurements of aging effective interaction potentials at the single cell level. They encapsulate information not only of the instantaneous stem cell-ligand interactions but also of their spatial and temporal evolution, bridging across scales. They specify the location, strength, and duration of these interactions. Furthermore, we show that the aging effective potential provides a previously unexplored route for extracting the change in configurational entropy of a living system, which allows the direct measurement of the organization of the environment by cells.

Through the comparison of experimental observations for mESCs with numerical simulations, we showed how short- and large-scale organization of beads, as measured by the effective potential and configurational entropy, emerge from the microscopic single cell-ligand interaction. We were able to distinguish three different mechanisms for cell-bead interaction. In the case of signaling Wnt beads, the interactions were dominated by a capturing dynamics, where cells encounter and retain beads in a seemingly irreversible manner, efficiently transporting them. In the case of nonsignaling iWnt beads, the reduction in the interactions was associated with a reduced cell-bead affinity, as if cells were disregarding a fraction of beads in their environment. Finally, the interactions between cells and Unc beads were found to be short-lived, with the lack of recruitment of these beads being crucial for reproducing the experimental phase diagram. To account for this observation in the numerical simulations, we allowed cells to interact with beads repeatedly. Combined with a high release rate, this method resulted in an effective pushing dynamics, where cells displace beads as they diffuse. The properties of the effective potential are robust to possible memory effects (see Fig. S9).

It is known that a suitable biochemical coating is necessary for the formation of durable cell-bead bonds $[30,31]$. Here, we have shown that the signaling nature of the coating plays a more complex role in these interactions. This role ranges from the recruiting dynamics to the location of the signal on the cell membrane. In the particular setup considered here, mESCs show a higher affinity for signaling (Wnt3a-coated) Wnt beads compared to the nonsignaling iWnt beads. Moreover, Wnt beads promote more effective interactions and organization by cells, over time, as seen in Fig. 1(c). These observations are in line with recent findings by Junyent et al. [7] on the molecular mechanisms that regulate the selectivity of ESCs to localized ligands. Those authors described a unique cross-talk between Wnt receptors and the activity of ionotropic glutamate receptors at mESC cytonemes, which contribute to the self-renewal of the mESC. It would be of great interest to determine whether this selectivity to particular signals is exclusive to embryonic stem cells or if it also applies to other cell types.

Through numerical simulations, we were able to explore regimes that provide deeper insight into the properties of the aging effective potential and the properties of cell-ligand interactions. We attributed the existence of a repulsive region in the phase diagram of the effective potential [such as Fig. 6(a)] to the active transport of ligands or beads by cells. Furthermore, we have shown how the transition of the potential, from attractive to repulsive, is directly related to the transport and displacement of ligands by the cells and is essential for recapitulating the experimental observations for Unc beads. The timing of this transition is controlled by the diffusive properties of cells, together with the microscopic (capture, disregard, and release) mechanisms of cell-ligand interactions. We expect that in experimental setups considering, for example, migratory phenotypes - such as cancer or skeletal stem cells - the repulsive phase will be observed even when cell-ligand interactions are dominated by a capturing mechanism.

By casting the configurational entropy of the system in terms of the effective potential, we could quantify the extent to which cells influence and organize their environment, as a function of time, and also as a function of the properties of the beads' coating and thus of the ligands. We observed that relevant ligands contribute to a faster organization of the environment [Figs. 8(a) and 9(b)]. Despite the similarities between the temporal evolution of the coordination number $c(t)$ [Fig. 4(a)] and the configurational entropy $s_{c}$ [Fig. 8(a)], the nature of both observables is 
different. The former is a measure only of the net accumulation of beads in a specific range of the effective potential, while the latter incorporates the whole spatial information of the effective potential, taking into account the structure and organization of the bead distribution. The lack of organization of Unc beads explains why even when we observe an increase in the coordination number for Unc beads [Fig. 4(a)], we observe no significant decrease in the configuration entropy over time [Fig. 8(a)]. Moreover, in the case of both Wnt and iWnt beads, the coordination number $c(t)$ reaches a plateau at the 6- or 9-h mark, respectively. However, in both cases, the configurational entropy maintains a constant rate for the whole duration of the analysis. The fact that the entropy continues to decrease despite no further bead accumulation indicates that, as time passes, cells are actively positioning the beads they have recruited at preferred locations on the cell membrane, further decreasing the entropy of the system. These preferred locations correspond to the minima of the effective potentials of interactions. Although the biological implications of this observation lie beyond the scope of the present work, we may hypothesize that the beads' location might be controlled by Wnt receptors and downstream effectors, and the dynamics of the cellular membrane.

In this work, we experimentally measured the motility of cells, which we used to interpret the effective potential observations. However, other parameters present in the model, such as the release rate and disregarding probability, could not be measured directly is because the cells' boundaries could not be detected accurately due to the lack of a membrane marker, which was not used in order to avoid external influence on the cell behavior (see Fig. 2). As Wnt is covalently bound to the bead and hence does not diffuse away from the beads [19], the interactionsrecruitment, release, and disregarding - rely completely on direct cell-bead contact. To experimentally determine and differentiate disregarding and release requires high spatial resolution for a reliable detection of how cells and beads interact at the microscopic scale.

An advantage of the effective potential approach we introduced is that it does not rely on the precise detection of cell boundaries but rather on the location of the cell center, which is much easier to determine. The methods presented here are applicable to a large number of systems, including other types of cells and ligands, both in 2D and 3D environments, such as bioengineered scaffolds or in vivo conditions. These applications could provide clues to better understand the formation of specialized microenvironments in multicellular tissues, such as stem-cell niches, which rely on the precise spatial organization and localization of cells and biochemical cues.

\section{ACKNOWLEDGMENTS}

We would like to thank Andy Thomas and Nemar Porats for their tireless computing support. I. B. acknowledges the support of a CONICYT Ph.D scholarship (Chile), Beca de Doctorado en el Extranjero No. 72160465. Furthermore, G.P. and I. B. acknowledge the support of the Centre for Doctoral Training on Theory and Simulation of Materials at Imperial College London, EPSRC (Grant No. EP/ L015579/1). This work was supported by a Sir Henry Dale fellowship (102513/Z/13/Z) to S. J. H.

I. B. and G. P. developed the methods for data analysis and the theoretical model. I. B. carried out analytic calculations and numerical simulations with the help of discussions with G.P., S.J.H. conceived the project and designed the experiment with the help of discussions with C. G., I. B., and G. P., C. G. and I. B. performed and optimized experiments. I. B. wrote the manuscript. All authors discussed and interpreted the data and commented on the manuscript.

\section{APPENDIX: Methods}

\section{Cell diffusion}

In our simulations, at each time step, a cell moves a fixed step length $\ell$ in a random direction in the two-dimensional plane. In the continuum limit, the two-dimensional diffusion equation for the probability $p(\mathbf{r}, t)$ of finding a cell at position $\mathbf{r}$, at time $t$, is given by

$$
\partial_{t} p(\mathbf{r}, t)=D \nabla^{2} p(\mathbf{r}, t),
$$

where $D$ is the diffusion constant and $\nabla^{2}$ is the twodimensional Laplacian. The Green function of Eq. (A1) on the infinite plane $\mathbb{R}^{2}$ is given by

$$
p(\mathbf{r}, t)=\frac{1}{4 \pi D t} \exp \left(-\frac{|\mathbf{r}|^{2}}{4 D t}\right),
$$

from which we can calculate the expected mean-square displacement of a cell,

$$
\left\langle|\mathbf{r}(t)|^{2}\right\rangle=\int \mathrm{d} \mathbf{r}|\mathbf{r}|^{2} p(\mathbf{r}, t)=4 D t .
$$

On the other hand, the mean-square displacement of a cell performing $t$ steps of fixed length $\ell$ in random directions is

$$
\left\langle|\mathbf{r}(t)|^{2}\right\rangle=\ell^{2} t .
$$

Comparing Eqs. (A3) and (A4) gives the diffusion constant

$$
D=\frac{\ell^{2}}{4} \text {. }
$$

\section{a. Experimental diffusion coefficient}

From the experimental trajectories of single cells, we calculate the effective diffusion constant $D$ on the timescale $\Delta t$ as 


$$
D(\Delta t)=\frac{\left\langle r^{2}(\Delta t)\right\rangle}{4 \Delta t}
$$

where $\Delta t$ is the averaging time window considered for computing the mean-square displacement. Given the trajectory $\mathbf{r}_{i}(t)$ of the $i$ th cell, measured with temporal resolution $\delta$ (1 $\mathrm{min}$ in our experiments) at times $t=0, \delta, 2 \delta, \ldots, T$, we can estimate the mean-square displacement as a function of $\Delta t$, a multiple of $\delta$, for the cell ensemble as

$$
\begin{aligned}
\left\langle r^{2}\right\rangle= & \frac{1}{N(1+(T-\Delta t) / \delta)} \\
& \times \sum_{i=1}^{N} \sum_{n=0}^{(T-\Delta t) / \delta}\left\|\mathbf{r}_{i}(n \delta+\Delta t)-\mathbf{r}_{i}(n \delta)\right\|^{2},
\end{aligned}
$$

where $N$ is the total number of cells in the experiment [37].

\section{2. van Hove correlation function}

Given a collection of $K$ particles of a given species, with positions $\mathbf{r}_{i}(t)$ as a function of time $t$ and particle index $i$, the van Hove dynamic correlation function reads

$$
G(\mathbf{r}, \tau)=\frac{1}{K}\left\langle\sum_{i=1}^{K} \sum_{j=1}^{K} \delta\left[\mathbf{r}-\mathbf{r}_{j}(\tau)+\mathbf{r}_{i}(0)\right]\right\rangle,
$$

which measures the average density of relative positions $\mathbf{r}=\mathbf{r}_{j}(t)-\mathbf{r}_{i}(0)$ of any two particles $i$ and $j$ at time $t=0$ and time $t=\tau$, respectively [24]. The brackets $\langle\cdot\rangle$ indicate the ensemble average. The van Hove correlation function can naturally be split into two terms:

$$
G(\mathbf{r}, \tau)=G_{s}(\mathbf{r}, \tau)+G_{d}(\mathbf{r}, \tau),
$$

where $G_{s}$ and $G_{d}$ are the self and distinct terms, respectively. The self part

$G_{s}(\mathbf{r}, \tau)=\frac{1}{K}\left\langle\sum_{i=1}^{K} \delta\left[\mathbf{r}^{\prime}+\mathbf{r}-\mathbf{r}_{i}(\tau)\right] \delta\left[\mathbf{r}^{\prime}-\mathbf{r}_{i}(0)\right]\right\rangle$

describes the contributions to the correlations from measuring the same particle at both time points, $t=0$ and $t=\tau$. The distinct part reads

$G_{d}(\mathbf{r}, \tau)=\frac{1}{K}\left\langle\sum_{i=1}^{K} \sum_{\substack{j=1 \\ j \neq i}}^{K} \delta\left[\mathbf{r}^{\prime}+\mathbf{r}-\mathbf{r}_{j}(\tau)\right] \delta\left[\mathbf{r}^{\prime}-\mathbf{r}_{i}(0)\right]\right\rangle$,

which measures the contributions to the correlations from distinct particles $(j \neq i)$.

\section{a. Extension to two species: Cells and beads}

To extend the van Hove correlation function to account for a second species, we consider that of the $K$ particles$N$ correspond to cells and $M$ to beads-such that $K=N+M$. If $\mathbf{R}_{i}$ denotes the position of cell $i \in$ $\{1,2, \ldots, N\}$ at time $t$, and $\mathbf{r}_{j}(t+\tau)$ denotes the position of bead $j \in\{1,2, \ldots, M\}$ at time $t+\tau$, we define

$\hat{G}(\mathbf{r}, t, \tau)=\frac{1}{N}\left\langle\sum_{i=1}^{N} \sum_{j=1}^{M} \delta\left[\mathbf{r}-\mathbf{r}_{j}(t+\tau)+\mathbf{R}_{i}(t)\right]\right\rangle$.

This correlation function, as previously defined in Eq. (1), considers only distinct particles of different species. As a result, it possesses no self part. As discussed in the main text, aging is introduced by allowing an explicit dependence on time $t$ of the positions of cells and beads.

\section{Multiple particle tracking}

To extract the positional information of cells and beads from the bright-field time-lapse images over time, we built a custom multiparticle tracking algorithm (implemented in MATLAB R2018b, The MathWorks, Inc.). This algorithm allowed the high-throughput analysis of the interaction of thousands of cells and beads at 1-min resolution and the calculation of the effective potential of interactions, Eq. (3). Cells were identified using a Sobel [38] edge-detection algorithm (sobel method in MATLAB's edge function) and screened according to size to exclude small objects, e.g., beads and debris, and large objects, e.g., cell doublets and clusters. Beads appeared as nearly perfectly black objects and were detected by applying a Laplacian-of-Gaussian filter (log method in MATLAB's edge function) and an intensity threshold to identify lower intensity (dark) objects. The detected candidate objects were screened according to eccentricity and size. Frame-to-frame nearestneighbor association was then performed to construct the trajectories of cells and beads. Each trajectory was stored with the corresponding object properties: boundary, area, major and minor axis lengths, and eccentricity.

\section{Experimental effective potential}

The age-dependent radial distribution function $\hat{g}(r, t, \tau)$, Eq. (2), and effective potential, Eq. (3), were computed by making use of the position of the cells and beads obtained from the tracking algorithm (methods Sec. A 3) as follows: (i) We measured the distance from the position of each cell at time $t$ to the position of every bead at time $t+\tau$, and computed the density of distances as a function of $r$ by pooling the data obtained for all cell-to-bead distances, from every biological repeat [see Fig. 10(a)]. (ii) A null model, corresponding to the noninteracting system, was constructed by considering a uniform, independently random distribution of beads and constructing the corresponding density profile, as done in (i), based on the positions of 


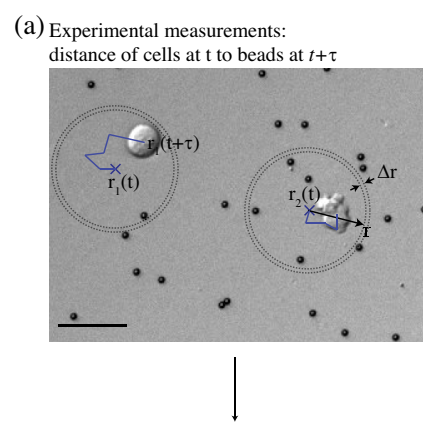

(b) Null model: uniform bead distribution
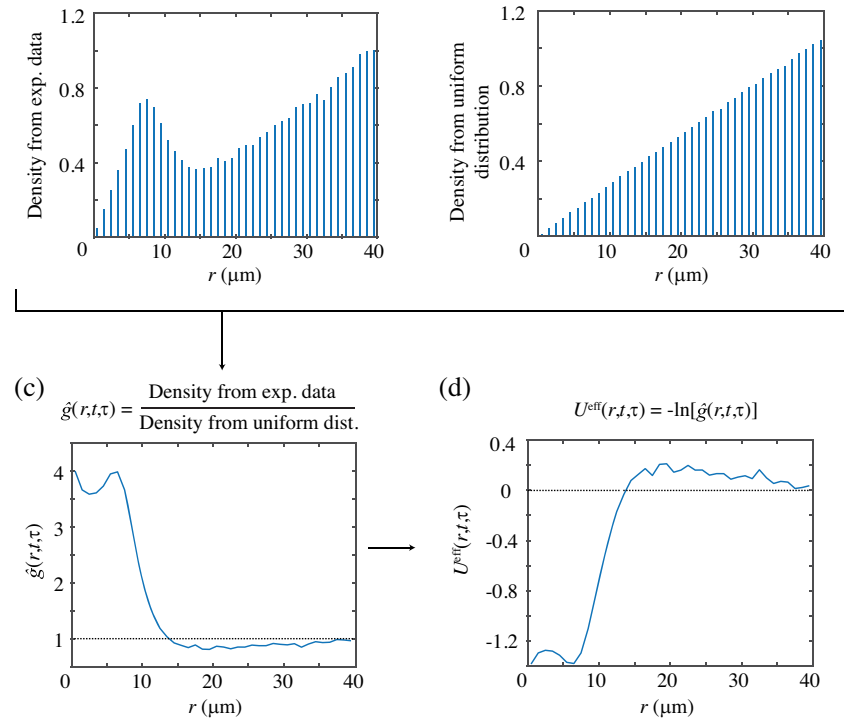

(d)

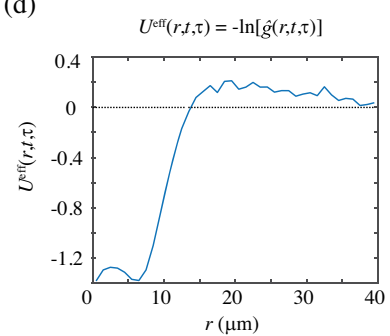

FIG. 10. Experimental measurement of the effective potential. Measurement of the distance from the position of every cell at time $t$ to the position of every bead at time $t+\tau$ (top) and the corresponding density of distances (bottom) for (a) the experimental positions of cells and beads and (b) the experimental positions of cells and uniformly distributed (numerically drawn) beads. Scale bars are set as $25 \mu \mathrm{m}$. (c) Radial distribution function measured as the ratio of the densities in panels (a) and (b). (d) Effective potential calculated as the negative logarithm of the radial distribution function in panel (c). Both $\hat{g}$ and $U^{\text {eff }}$ are dimensionless.

cells obtained experimentally [see Fig. 10(b)]. The uniform distribution of beads is constructed numerically and has the advantage of taking into account the finite size of the field of view, although these finite-size effects are noticeable only for large values of $r$. (iii) By computing the ratio between the experimental densities obtained in (i) and the reference densities in (ii), we obtain the $\operatorname{RDF} \hat{g}(r, t, \tau)$ [Fig. 10(c)]. (iv) Finally, the effective potential of interactions is obtained by taking the negative natural logarithm of the RDF, $U^{\text {eff }}=-\ln [\hat{g}(r, t, \tau)]$.

\section{a. Points of interest in the effective potential}

We define three points of interest in the effective potentials: (i) The first is the maximum accumulation point (MAP), defined as the minimum of the effective potential, where its spatial derivative vanishes, i.e., $\mathrm{d} U^{\text {eff }} / \mathrm{d} r=0$.
This point marks the most probable location of the beads along a radial line starting at $r=0$. This point is indicated by the symbol filled down-pointing triangle in Fig. 3. (ii) The second point is the maximum depletion point (MDP), defined as the maximum of the potential where $\mathrm{d} U^{\text {eff }} / \mathrm{d} r=0$. It marks the least-probable location of beads along the radial coordinate and is indicated by the symbol filled up-pointing triangle in Fig. 3. (iii) The range of interactions is defined as the first point beyond both the MAP and MDP where $U^{\mathrm{eff}}(r, t)=0$. This point measures the effective distance up to which cells deplete the environment of beads. The range is indicated by filled circle in Fig. 3.

\section{Solution of the model with release}

The master equation of the probability $P_{\nu}(n, t)$ to find $n$ beads in contact with a cell at time $t$, as beads can be released by the cell at constant rate $\nu$, is given by Eq. (13). We consider the initial condition $P_{\nu}(n, t=0)=\delta_{n, 0}$. To solve it, we introduce a generating function

$$
\phi_{\nu}(z, t)=\sum_{n=0}^{\infty} z^{n} P_{\nu}(n, t)
$$

with normalization

$$
\phi_{\nu}(1, t)=\sum_{n=0}^{\infty} P(n, t)=1 .
$$

Using Eq. (A13), Eq. (13) produces a first-order, linear partial differential equation for $\phi_{\nu}(z, t)$,

$\frac{1}{1-z} \frac{\partial \phi_{\nu}(z, t)}{\partial t}-\nu \frac{\partial \phi_{\nu}(z, t)}{\partial z}=-\rho_{b} \frac{d A(t)}{d t} \phi_{\nu}(z, t)$,

where $A(0)=0$. Equation (A15) can be solved by the method of characteristics [39]. Introducing

$$
\eta=\eta(z, t ; \nu)
$$

we demand that $\phi_{\nu}=w(\eta(z, t ; \nu), t)$ with a hitherto unknown function $w$ in two variables. Equation (A15) can now be written in terms of $w$,

$\frac{\partial w}{\partial t}+\frac{\partial w}{\partial \eta}\left(\frac{\partial \eta}{\partial t}-(1-z) \nu \frac{\partial \eta}{\partial z}\right)+(1-z) \rho_{b} \dot{A}(t) w=0$

Demanding

$$
\frac{\partial \eta}{\partial t}-(1-z) \nu \frac{\partial \eta}{\partial z}=0
$$

such that, for example, 


$$
\eta(z, t ; \nu)=\ln (1-z)-\nu t
$$

means that Eq. (A17) reduces to

$$
\frac{\partial w}{\partial t}+(1-z) \rho_{b} \dot{A}(\xi) w=0,
$$

which can immediately be solved to produce

$$
w(\eta, t)=C(\eta) \exp \left(-\int^{t} \mathrm{~d} s(1-z) \rho_{b} \dot{A}(s)\right) .
$$

Noticing that $1-z$ is given in terms of $\eta$ and $t$ by Eq. (A19), we obtain

$$
w(\eta, t)=C(\eta) \exp \left(-\rho_{b} \mathrm{e}^{\eta} \int_{0}^{t} \mathrm{~d} s \mathrm{e}^{\nu s} \dot{A}(s)\right) .
$$

By writing Eq. (A22) in terms of $z$ and $t$, and imposing the normalization Eq. (A14), which implies $C=1$, we find

$\phi_{\nu}(z, t)=\exp \left(\rho_{b}(z-1)\left[\int_{0}^{t} \mathrm{~d} s \mathrm{e}^{-\nu(t-s)} \dot{A}(s)\right]\right)$.

Finally, we obtain the probability $P_{\nu}(n, t)$ of finding $n$ beads attached to a cell at time $t$, as the coefficients of $n$ th-order term in the Taylor expansion of Eq. (A23) around $z=0$. Thus, we get

$$
P_{\nu}(n, t)=\frac{\alpha^{n}(A, \nu, t)}{n !} e^{-\alpha(A, \nu, t)},
$$

where $\alpha(A, \nu, t)=\rho_{b} \int_{0}^{t} \mathrm{~d} s e^{-\nu(t-s)} \dot{A}(s)$ is the time-dependent expected number of beads attached to the cell, as discussed in the main text.

[1] M. Thomson, S. J. Liu, L.-N. Zou, Z. Smith, A. Meissner, and S. Ramanathan, Pluripotency Factors in Embryonic Stem Cells Regulate Differentiation into Germ Layers, Cell 145, 875 (2011).

[2] A. J. Engler, S. Sen, H. L. Sweeney, and D. E. Discher, Matrix Elasticity Directs Stem Cell Lineage Specification, Cell 126, 677 (2006).

[3] M. Zecca, K. Basler, and G. Struhl, Direct and Long-Range Action of a Wingless Morphogen Gradient, Cell 87, 833 (1996).

[4] S. Kondo and T. Miura, Reaction-Diffusion Model as a Framework for Understanding Biological Pattern Formation, Science 329, 1616 (2010).

[5] P. Müller, K. W. Rogers, R. Yu. Shuizi, M. Brand, and A. F. Schier, Morphogen Transport, Development 140, 1621 (2013).

[6] A. M. Pani and B. Goldstein, Direct Visualization of a Native Wnt In Vivo Reveals that a Long-Range Wnt
Gradient Forms by Extracellular Dispersal, eLife 7, e38325 (2018).

[7] S. Junyent, C. L. Garcin, J. L. A. Szczerkowski, T.-J. Trieu, J. Reeves, and S. J. Habib, Specialized Cytonemes Induce Self-Organization of Stem Cells, Proc. Natl. Acad. Sci. U.S.A. 117, 7236 (2020).

[8] U. Cavallaro and G. Christofori, Cell Adhesion and Signalling by Cadherins and Ig-CAMs in cancer, Nat. Rev. Cancer 4, 118 (2004).

[9] S. J. Bray, Notch Signalling: A Simple Pathway Becomes Complex, Nat. Rev. Mol. Cell Biol. 7, 678 (2006).

[10] R. Nusse and H. Clevers, Wnt/ $\beta$-Catenin Signaling, Disease, and Emerging Therapeutic Modalities, Cell 169, 985 (2017).

[11] C. Alexandre, A. Baena-Lopez, and J.-P. Vincent, Patterning and Growth Control by Membrane-Tethered Wingless, Nature (London) 505, 180 (2014).

[12] M. C. Marchetti, J.-F. Joanny, S. Ramaswamy, T. B. Liverpool, J. Prost, M. Rao, and R. A. Simha, Hydrodynamics of Soft Active Matter, Rev. Mod. Phys. 85, 1143 (2013).

[13] U. M. B. Marconi, M. Paoluzzi, and C. Maggi, Effective Potential Method for Active Particles, Mol. Phys. 114, 2400 (2016).

[14] T. E. Angelini, E. Hannezo, X. Trepat, M. Marquez, J. J. Fredberg, and D. A. Weitz, Glass-like Dynamics of Collective Cell Migration, Proc. Natl. Acad. Sci. U.S.A. 108, 4714 (2011).

[15] K. Kawaguchi, R. Kageyama, and M. Sano, Topological Defects Control Collective Dynamics in Neural Progenitor Cell Cultures, Nature (London) 545, 327 (2017).

[16] S. Garcia, E. Hannezo, J. Elgeti, J.-F. Joanny, P. Silberzan, and N. S. Gov, Physics of Active Jamming During Collective Cellular Motion in a Monolayer, Proc. Natl. Acad. Sci. U.S.A. 112, 15314 (2015).

[17] D. Chen, W. Y. Aw, D. Devenport, and S. Torquato, Structural Characterization and Statistical-Mechanical Model of Epidermal Patterns, Biophys. J. 111, 2534 (2016).

[18] S. J. Habib, B.-C. Chen, F.-C. Tsai, K. Anastassiadis, T. Meyer, E. Betzig, and R. Nusse, A Localized Wnt Signal Orients Asymmetric Stem Cell Division In Vitro, Science 339, 1445 (2013).

[19] M. Lowndes, S. Junyent, and S. J. Habib, Constructing Cellular Niche Properties by Localized Presentation of Wnt Proteins on Synthetic Surfaces, Nat. Protoc. 12, 1498 (2017).

[20] B. Ma, T.-J. Trieu, J. Cheng, S. Zhou, Q. Tang, J. Xie, J.-L. Liu, K. Zhao, S. J. Habib, and X. Chen, Differential Histone Distribution Patterns in Induced Asymmetrically Dividing Mouse Embryonic Stem Cells, Cell Reports 32, 108003 (2020).

[21] H. F. Farin, I. Jordens, M. H. Mosa, O. Basak, J. Korving, D. V. F. Tauriello, K. de Punder, S. Angers, P. J. Peters, M. M. Maurice et al., Visualization of a Short-Range Wnt Gradient in the Intestinal Stem-Cell Niche, Nature (London) 530, 340 (2016).

[22] R. Raz, C.-K. Lee, L. A. Cannizzaro, P. d'Eustachio, and D. E. Levy, Essential Role of STAT3 for Embryonic Stem Cell Pluripotency, Proc. Natl. Acad. Sci. U.S.A. 96, 2846 (1999). 
[23] D. T. Berge, D. Kurek, T. Blauwkamp, W. Koole, A. Maas, E. Eroglu, R. K. Siu, and R. Nusse, Embryonic Stem Cells Require Wnt Proteins to Prevent Differentiation to Epiblast Stem Cells, Nat. Cell Biol. 13, 1070 (2011).

[24] J.-P. Hansen and I. R. McDonald, Theory of Simple Liquids: With Applications to Soft Matter (Academic Press, New York, 2013).

[25] M. Henkel and M. Pleimling, Non-Equilibrium Phase Transitions: Volume 2: Ageing and Dynamical Scaling Far from Equilibrium (Springer, New York, 2011).

[26] A. Ben-Naim, Statistical Potentials Extracted from Protein Structures: Are These Meaningful Potentials?, J. Chem. Phys. 107, 3698 (1997).

[27] T. Hamelryck, M. Borg, M. Paluszewski, J. Paulsen, J. Frellsen, C. Andreetta, W. Boomsma, S. Bottaro, and J. Ferkinghoff-Borg, Potentials of Mean Force for Protein Structure Prediction Vindicated, Formalized and Generalized, PloS One 5, e13714 (2010).

[28] See Supplemental Material at http://link.aps.org/ supplemental/10.1103/PhysRevX.10.041022 for Supplemental Figs. S1-S9.

[29] U. Hersel, C. Dahmen, and H. Kessler, RGD Modified Polymers: Biomaterials for Stimulated Cell Adhesion and Beyond, Biomaterials 24, 4385 (2003).

[30] A. E. Nel, L. Mädler, D. Velegol, T. Xia, E. M. V. Hoek, P. Somasundaran, F. Klaessig, V. Castranova, and M. Thompson, Understanding Biophysicochemical Interactions at the Nano-Bio Interface, Nat. Mater. 8, 543 (2009).
[31] T. Patiño, J. Soriano, L. Barrios, E. Ibáñez, and C. Nogués, Surface Modification of Microparticles Causes Differential Uptake Responses in Normal and Tumoral Human Breast Epithelial Cells, Sci. Rep. 5, 11371 (2015).

[32] D. Chandler, Introduction to Modern Statistical Mechanics (Oxford University Press, New York, 1987).

[33] A. M. Berezhkovskii, Yu. A. Makhnovskii, and R. A. Suris, Wiener Sausage Volume Moments, J. Stat. Phys. 57, 333 (1989).

[34] S. Nekovar and G. Pruessner, A Field-Theoretic Approach to the Wiener Sausage, J. Stat. Phys. 163, 604 (2016).

[35] M. Chupeau, O. Bénichou, and R. Voituriez, Cover Times of Random Searches, Nat. Phys. 11, 844 (2015).

[36] H. J. Raveché, Entropy and Molecular Correlation Functions in Open Systems. I. Derivation, J. Chem. Phys. 55, 2242 (1971).

[37] E. Kepten, A. Weron, G. Sikora, K. Burnecki, and Y. Garini, Guidelines for the Fitting of Anomalous Diffusion Mean Square Displacement Graphs from Single Particle Tracking Experiments, PLoS One 10, e0117722 (2015).

[38] O. R. Vincent and O. Folorunso, A Descriptive Algorithm for Sobel Image Edge Detection, in Proceedings of Informing Science \& IT Education Conference (InSITE), volume 40, page 97, 2009.

[39] R. Courant and D. Hilbert, Methods of Mathematical Physics: Partial Differential Equations (John Wiley \& Sons, New York, 1962), Vol. II. 